\title{
The infinitesimal phase response curves of oscillators in piecewise smooth dynamical systems $\dagger$
}

\author{
Y. PARK ${ }^{1,2}$, K. M. SHAW ${ }^{3,4}$, H. J. CHIEL ${ }^{3}$ and P. J. THOMAS ${ }^{1}$ \\ ${ }^{1}$ Department of Mathematics, Applied Mathematics, and Statistics, Case Western Reserve University, Cleveland, \\ OH 44106, USA \\ email:yop6@pitt.edu,pjthomas@case.edu \\ ${ }^{2}$ Department of Mathematics, University of Pittsburgh, Pittsburgh, PA 15260, USA \\ ${ }^{3}$ Department of Biology, Case Western Reserve University, Cleveland, OH 44106, USA \\ e-mail:hjc@case.edu \\ ${ }^{4}$ Department of Anesthesia, Critical Care, Small and Pain Medicine, Massachusetts General Hospital, \\ Boston, MA 02114, USA \\ email:kmshaw@partners.org
}

(Received 28 July 2017; revised 28 February 2018; accepted 1 March 2018; first published online 2 April 2018)

\begin{abstract}
The asymptotic phase $\theta$ of an initial point $x$ in the stable manifold of a limit cycle (LC) identifies the phase of the point on the LC to which the flow $\phi_{t}(x)$ converges as $t \rightarrow \infty$. The infinitesimal phase response curve (iPRC) quantifies the change in timing due to a small perturbation of a LC trajectory. For a stable LC in a smooth dynamical system, the iPRC is the gradient $\nabla_{x}(\theta)$ of the phase function, which can be obtained via the adjoint of the variational equation. For systems with discontinuous dynamics, the standard approach to obtaining the iPRC fails. We derive a formula for the iPRCs of LCs occurring in piecewise smooth (Filippov) dynamical systems of arbitrary dimension, subject to a transverse flow condition. Discontinuous jumps in the iPRC can occur at the boundaries separating subdomains, and are captured by a linear matching condition. The matching matrix, $M$, can be derived from the saltation matrix arising in the associated variational problem. For the special case of linear dynamics away from switching boundaries, we obtain an explicit expression for the iPRC. We present examples from cell biology (Glass networks) and neuroscience (central pattern generator models). We apply the iPRCs obtained to study synchronization and phase-locking in piecewise smooth LC systems in which synchronization arises solely due to the crossing of switching manifolds.
\end{abstract}

Key words: Mathematical biology, Nonsmooth analysis, Phase plane analysis, Nonlinear oscillators

\section{Introduction}

\subsection{Overview}

A stable limit cycle (LC) is a closed, isolated periodic orbit in a nonlinear dynamical system that attracts nearby trajectories [22]. LCs arise in models of biological motor control systems [25,30], excitable membranes [13,27], sensory systems [18], neuropathologies

$\dagger$ This work was supported in part by NSF grant DMS-1413770 and NSF grant DMS-1010434. 
such as Parkinsonian tremor [41] and epilepsy [58]. Chemical oscillations arise when the differential equations describing mass action kinetics admit a LC [16]. LC dynamics appear not only in biological but also in engineered systems. For instance, phase locked loops play a role in radio and electronic communications devices [59], and control of oscillations is an important problem in mechanical and electrical engineering [52].

Formally, a nonlinear autonomous $n$-dimensional ordinary differential equation

$$
\frac{d}{d t} \boldsymbol{x}(t)=\boldsymbol{F}(\boldsymbol{x}(t))
$$

has a stable $T$-periodic LC if it admits a periodic solution $\gamma$ with minimal period

$$
\gamma(t)=\gamma(t+T), \quad \forall t \in \mathbb{R},
$$

and an open neighbourhood of $\gamma$ (the basin of attraction, B.A.) within which all initial conditions give solutions converging, as $t \rightarrow \infty$, to the set

$$
\Gamma=\{\gamma(s): s \in[0, T)\} .
$$

In many situations, the multidimensional dynamics of a stable LC oscillator can be accurately captured in a one-dimensional phase model, representing the fraction of progress around the LC. The effect of weak inputs on the oscillator can be represented in terms of their effect on the timing of the LC alone, the linear approximation to which is the infinitesimal phase response curve (iPRC). The iPRC has become a fundamental tool for understanding entrainment and synchronization phenomena in weakly driven and weakly coupled oscillator systems, respectively [14,53]. The iPRC gives the relative shift in timing per unit stimulus, as a function of the phase at which the stimulus occurs, in the limit of small stimulus size (Figure 1B; [14]). The iPRC $z(t)$ is a vector quantity; it is equivalent to the gradient of the asymptotic phase function $\theta:$ B.A. $\rightarrow[0,1)$. The phase function $\theta$ maps each point in the basin of attraction to a point on the circle labelling the LC trajectory to which it converges as $t \rightarrow \infty$. In particular, the labelling is simply chosen to be $\theta=t / T \bmod T$. A displacement from the LC by amount $\Delta y$ at time $t \in[0, T)$ causes a shift in timing equal to $T \Delta \boldsymbol{y} \cdot \boldsymbol{z}(t)+o(|\Delta \boldsymbol{y}|)$, in the limit as $|\Delta \boldsymbol{y}| \rightarrow 0$.

The iPRC is known in closed form in a handful of special cases: near a supercritical Andronov-Hopf bifurcation [27], near a saddle-node-on-invariant-circle (SNIC) bifurcation [4], and for certain piecewise linear (PWL) oscillator models [8, 10,55]. The form of the iPRC near a homoclinic bifurcation is not known in general, cf. $[4,35,55]$.

For general smooth nonlinear systems with LC dynamics, one may obtain the iPRC numerically using an adjoint method [28] or via continuation of a two-point boundary value problem [45]. If oscillations arise from a dynamical system (1.1), where $\boldsymbol{F}: \mathbb{R}^{n} \rightarrow \mathbb{R}^{n}$ is a $C^{1}$ differentiable map, then the iPRC is a $T$-periodic vector function of time that obeys an adjoint equation

$$
d z / d t=A(t) z(t), \quad A(t)=-(D F(\gamma(t)))^{\top},
$$

together with the boundary condition $z(t)=z(t+T)$. The 'adjoint operator' $A(t)$ is the transpose of the negative Jacobian matrix $D F$ evaluated at the LC $\gamma(t)[14,27]$. 
(a)
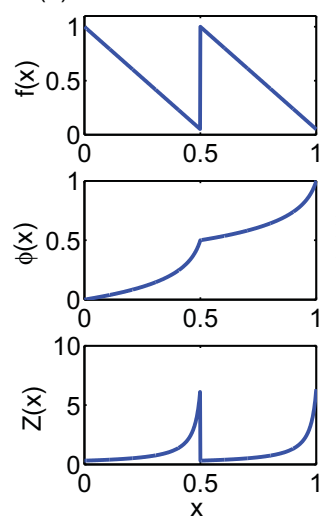
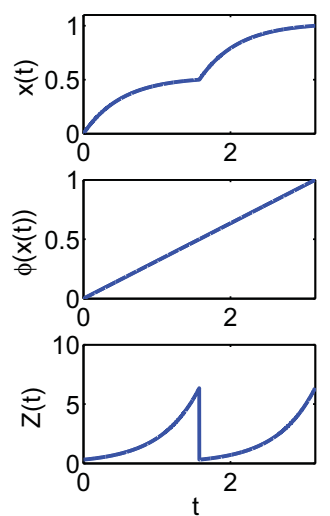

(b)

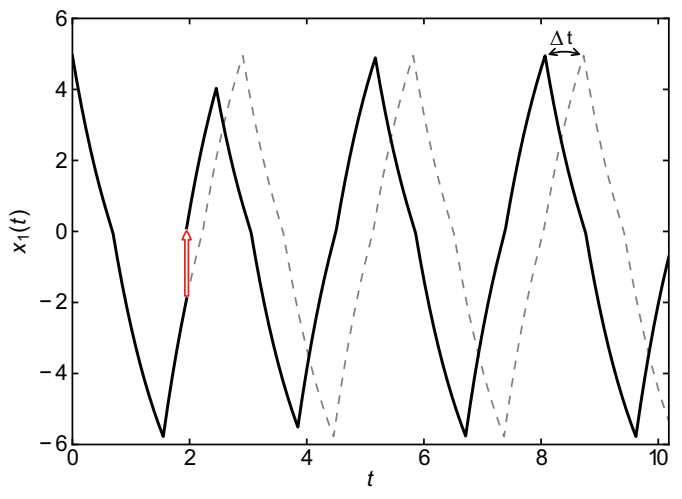

FIGURE 1. 1-D oscillator with piecewise smooth velocity. (A) Top-left: Velocity $f(x)=1-2 \alpha x$ for $0 \leqslant x<1 / 2$ and $f(x)=1-2 \alpha(x-1 / 2)$ for $1 / 2 \leqslant x<1$, with $\alpha=0.95$. The period is $T=\frac{1}{\alpha} \ln \left(\frac{1}{1-\alpha}\right) \approx 3.153$. Top-right: trajectory $x(t)$. Center: oscillator phase $\phi$ plotted versus space $(x$, left $)$ and versus time ( $t$, right). Bottom: infinitesimal phase response curve $Z$ plotted versus space ( $x$, left) and versus time (t, right). The phase $\phi$ is a continuous mapping from $x$ to the circle $[0,1)$. The iPRC jumps by $-\frac{\alpha^{2}}{1-\alpha}\left[\ln \frac{1}{1-\alpha}\right]^{-1} \approx-6.025$ at $x=1 / 2$ and again at $x=0$. (B) Direct perturbation (red arrow) and phase response $(\Delta \theta)$ for a limit cycle solution of a 2-D Glass network model. For a perturbation of size $\varepsilon$, we recover the iPRC value as $\lim _{\varepsilon \rightarrow 0} \Delta \theta / \varepsilon$ [14]. The $\Delta t$ in the figure is equivalent to $\Delta \theta T$, where $T$ is the oscillator period.

Both the adjoint equation method and the continuation-based method break down for nonsmooth systems, i.e., for systems such that the Jacobian DF is not defined at all points around the LC. Such cases arise in piecewise smooth systems, where the vector field changes abruptly across some boundary $\Sigma$. The monograph [11] classifies piecewise smooth systems according to the degree of smoothness at the boundaries. If across a boundary $\Sigma$, the vector fields $F_{1}, F_{2}$ are discontinuous $\left(F_{1}(\mathbf{x}) \neq F_{2}(\mathbf{x})\right)$ at a point $\mathbf{x} \in \Sigma$, then the system is said to have degree of smoothness one, and are said to be of Filippov type (also called differential inclusions [17]; the derivative of a solution passing through such a point may take a value in some well-defined set, rather than equalling a unique value). If the vector fields across the boundary satisfy $F_{1}(\mathbf{x})=F_{2}(\mathbf{x})$, but differ in their Jacobians $\left(D F_{1} \neq D F_{2}\right)$ at $\mathbf{x}$, then the degree of smoothness is said to be 2 . This definition generalizes to higher order derivatives in a natural way. Systems with smoothness degree two or higher are called piecewise-smooth continuous systems ([11], p. 74). Finally, a system with a discontinuous solution at the boundary $\Sigma$ is said to have degree of smoothness zero. In this paper, we consider systems with smoothness one or higher, i.e., we assume the solutions are continuous functions of time.

If $F$ is either a Filippov system or piecewise smooth continuous system, the Jacobian linearization may break down at the boundaries separating the regions within which the vector field is smooth. The theory of isochrons, and the subsequent iPRC, is well developed for smooth systems [21], with considerably less literature for iPRCs in nonsmooth differential equations. Infinitesimal PRCs have been computed explicitly in some planar systems $[8,55]$. Recent literature shows a significant interest in the analysis of iPRCs for 
piecewise smooth systems in both biological and control engineering contexts $[7,10,26,56]$. We compare these studies in detail in Section 4.2.

Nonsmooth oscillator models arise in both biological and engineered systems. Examples include planar nonlinear integrate-and-fire neural models [10], PWL approximations to the Hindmarsch-Rose neural model [50], and models of anti-lock braking systems [43,49]. Section Section 4.4 mentions additional examples. Existence of oscillatory solutions in piecewise smooth systems is a question of interest in its own right $[17,24,36,43,62]$.

In this paper, we derive a formula for the iPRC of stable LCs that allows for discontinuities at the domain boundaries of piecewise smooth dynamical systems. In the case of PWL systems, we obtain an explicit expression in terms of the system coefficients for each subdomain through which the LC travels, and the tangent vectors of the surfaces separating the regions where the vector field definition changes (equation (2.19) in Section 2.2). To obtain these results, we derive a jump condition satisfied by the iPRC at the boundaries between subdomains.

Overview of the paper: In the next Section 1.2, we develop a motivating one-dimensional example in detail. In Section 2, we calculate the form of the discontinuity in the iPRC for a LC in a piecewise smooth dynamical system in arbitrary dimensions. In the case of LCs arising in $n$-dimensional PWL dynamical systems, we provide an explicit closed form for the iPRC. Section 2.1 lays out the assumptions needed to establish our results, and Section 2.2 presents the main Theorem (2.1) giving the correction to the iPRC upon crossing a switching boundary. Section 3 provides examples of iPRCs in nonsmooth systems: a planar piecewise constant system where the nonlinearities arise strictly from the boundaries in Section 3.1, a planar PWL oscillator introduced in a motor control context [55], but generalized here to a non-symmetric geometry in Section 3.2, a PWL genetic regulatory circuit model (Glass network [19,20]) in Section 3.3, a three-dimensional motor control model [54] in Section 3.4, a four-dimensional weakly diffusively coupled version of the piecewise constant system in Section 3.4.1, and a six-dimensional threshold linear network model comprising of two weakly coupled three-dimensional oscillators [42] in Section 3.4.2. In Section 4.1, we discuss the relation between our boundary-crossing correction matrix and the classical saltation matrix, in Section 4.2, we discuss the limitations of the method, and in Section 4.3, we discuss a range of possible further applications. Following our conclusion Section 5, the appendices detail the proofs and derivations of the results.

The main results reported here appeared previously in the Master's thesis of the first author [46].

\subsection{A 1-D example}

To illustrate the necessity of including a jump condition in the phase response for piecewise smooth systems, consider the following one-dimensional example (see also $[7,26]$ ).

Let $f_{1}$ and $f_{2}$ be smooth, strictly positive functions defined on the unit interval. Identify the interval with the circle and let $x \in[0,1)$ evolve according to

$$
\frac{d x}{d t}=\left\{\begin{array}{l}
f_{1}(x), 0 \leqslant x<a \\
f_{2}(x), a \leqslant x<1
\end{array}\right.
$$


where $0<a<1$ marks the location at which the rate law for $x$ changes from $f_{1}$ to $f_{2}$. The rate law changes back to $f_{1}$ when $x$ wraps around from one to zero again. The period of this oscillator is

$$
T=\int_{0}^{a} \frac{d x}{f_{1}(x)}+\int_{a}^{1} \frac{d x}{f_{2}(x)} .
$$

We can define a phase variable $\phi(x)$ by the condition $d \phi / d t=1 / T$, which gives the form

$$
\phi(x)-\phi(0)=\left\{\begin{array}{r}
\frac{1}{T} \int_{0}^{x} \frac{d x^{\prime}}{f_{1}\left(x^{\prime}\right)}, 0 \leqslant x \leqslant a \\
\phi(a)+\frac{1}{T} \int_{a}^{x} \frac{d x^{\prime}}{f_{2}\left(x^{\prime}\right)}, a<x \leqslant 1 .
\end{array}\right.
$$

Here, $\phi(0)$ is an arbitrary constant that we may set to zero, without loss of generality. The iPRC $Z_{x}$ for this system describes the shift in timing of the oscillation upon making a small displacement in the $x$ coordinate, as a function of position. The iPRC is

$$
Z_{x}=\frac{d \phi}{d x}=\left\{\begin{array}{l}
Z_{1} \equiv\left(T f_{1}(x)\right)^{-1}, 0 \leqslant x<a \\
Z_{2} \equiv\left(T f_{2}(x)\right)^{-1}, a<x \leqslant 1
\end{array}\right.
$$

The iPRC has a finite jump discontinuity at the location $a$ where the rate law for $x$ changes, namely

$$
Z_{2}(a)-Z_{1}(a)=\frac{1}{T}\left(\frac{1}{f_{2}(a)}-\frac{1}{f_{1}(a)}\right) .
$$

As a specific example, consider the rate laws $f_{1}(x)=1-2 \alpha x, f_{2}(x)=1-2 \alpha(x-1 / 2)$, parameterized by $\alpha<1$, with switching point $a=1 / 2$. For this example, $T=\frac{1}{\alpha} \ln \left(\frac{1}{1-\alpha}\right)$, $\phi(x)=\frac{-1}{2 \ln (1-\alpha)} \ln \left(\frac{1}{1-2 \alpha x}\right)$ for $0 \leqslant x \leqslant 1 / 2$, and $\phi(x)=\frac{1}{2}-\frac{1}{2 \ln (1-\alpha)} \ln \left(\frac{1}{1-2 \alpha(x-1 / 2)}\right)$ for $1 / 2 \leqslant x<1$. The iPRC in the first and second intervals are $Z_{1}(t)=\frac{\alpha}{1-2 \alpha x(t)}\left[\ln \frac{1}{1-\alpha}\right]^{-1}$ and $Z_{2}(t)=\frac{\alpha}{1-2 \alpha(x(t)-1 / 2)}\left[\ln \frac{1}{1-\alpha}\right]^{-1}$, respectively. The phase $\phi(x)$ is continuous across the switch points $x=1 / 2$ and $x=0$. The jump in the iPRC is $Z_{2}\left(T_{a}\right)-Z_{1}\left(T_{a}\right)=-\frac{\alpha^{2}}{1-\alpha}\left[\ln \frac{1}{1-\alpha}\right]^{-1}$; here $T_{a}=T / 2$ is the time at which the trajectory reaches the switching point $a=1 / 2$. Figure 1 illustrates this scenario for $\alpha=0.95$.

We remark that the size of the discontinuity in this example could be computed explicitly because the domain is one-dimensional, and the normalization condition $d \phi / d t=1 / T$ yields one equation with one unknown for the size of the discontinuity. However, in $n>1$ dimensions, the normalization condition only yields one equation in $n$ unknowns. In the following section, we derive the remaining $n-1$ equations required to determine the size of the discontinuity in the general case.

\section{Methods}

\subsection{Definitions and hypotheses required to solve the adjoint equation over differential inclusions}

We introduce notation needed to discuss iPRCs for differential inclusion systems. For a general treatment of Filippov systems, see [17]. 
Limit cycles and asymptotic phase. In a smooth system of the form (1.1), possessing a stable LC $\gamma$, we associate a phase $\theta \in[0,1)$ with points along the cycle such that

$$
d \theta(\gamma(t)) / d t=1 / T
$$

where $T$ is the period of the LC and $\theta\left(\gamma\left(t_{0}\right)\right)=0$ is chosen arbitrarily. To each point $\boldsymbol{x}_{0}$ in the basin of attraction (B.A.) we assign a phase $\theta\left(x_{0}\right) \in[0,1)$ such that the trajectory $\boldsymbol{x}(t)$ with initial condition $\boldsymbol{x}(0)=\boldsymbol{x}_{0}$ satisfies

$$
\lim _{t \rightarrow \infty}\left\|\boldsymbol{x}(t)-\gamma\left(t+T \theta\left(\boldsymbol{x}_{0}\right)\right)\right\| \rightarrow 0 .
$$

The isochrons are level curves of the phase function $\theta\left(\boldsymbol{x}_{0}\right)$, and foliate the basin of attraction. For a stable LC in a smooth dynamical system, the existence of the phase function is a well-known consequence of results from invariant manifold theory [21]. Intuitively, isochrons indicate which points in the basin of attraction eventually converge to the LC solution having a particular phase.

Filippov systems. Let $D$ be a path connected subset of $\mathbb{R}^{n}$. We say that an autonomous vector field $\boldsymbol{F}: D \rightarrow \mathbb{R}^{n}$ is piecewise smooth on $D$ if there exist a finite number, $R$, of open sets $D_{r}$ such that the following hypotheses hold:

(H1) $D_{r}$ is nonempty, simply connected, and open for each $r$.

(H2) $D_{i} \cap D_{j}=\varnothing, \forall i \neq j$.

(H3) $D \subset \bigcup_{r=1}^{R} \bar{D}_{r}$.

(H4) There exist $C^{1}$, bounded vector fields $\boldsymbol{F}_{r}: \bar{D}_{r} \rightarrow \mathbb{R}^{n}$ such that for all $x$ in $D_{r}$, $\boldsymbol{F}_{r}(x)=\boldsymbol{F}(x)$.

Note that we require $\boldsymbol{F}_{r}=\boldsymbol{F}$ only on the interior of each open domain $D_{r}$, while we require that each $\boldsymbol{F}_{r}$ be smooth on the closure $\bar{D}_{r}$.

The corresponding dynamical system

$$
\frac{d \boldsymbol{x}}{d t}=\boldsymbol{F}(\boldsymbol{x})
$$

is called a piecewise smooth dynamical system or a Filippov system [17].

We further restrict our attention to Filippov systems satisfying the following assumptions:

(1) The system (2.3) has a stable T-periodic limit cycle (LC) that crosses each boundary transversely with nonzero speed. Because the LC could cross the same boundary multiple times, we introduce a separate label for each segment of the LC lying between two boundary crossings (Figure 2). Thus, we label each piecewise smooth portion of the LC by a number $k=1, \ldots, K$ (see equation (2.9)), and use the same convention of the regions. The boundary between the $k$ th and $(k+1)$-st portions of the LC (between regions $D_{k}$ and $D_{k+1}$ ) is a surface denoted $\Sigma_{k+1}$. We denote the point at which the LC crosses this boundary by $\boldsymbol{p}_{k+1} \in \Sigma_{k+1}$.

(2) Each boundary is at least $C^{1}$ in an open ball $B\left(\boldsymbol{p}_{k+1}, c\right)$, centered at $\boldsymbol{p}_{k+1}$ with radius c. From this assumption, it follows that at each crossing point $\boldsymbol{p}_{k+1}$ there exists a tangent hyperplane spanned by $n-1$ orthonormal basis vectors, denoted $\hat{\boldsymbol{w}}_{i}^{k+1}$ for 


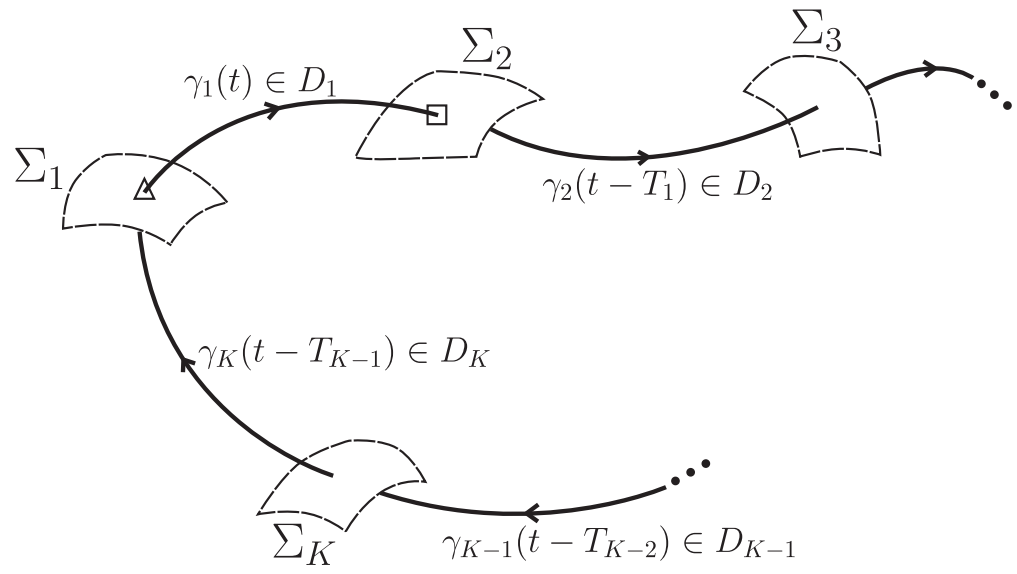

FiguRE 2. Sections of the piecewise smooth dynamical system. Full domain boundaries are omitted for clarity. The first segment of the limit cycle begins at point $\gamma_{1}(0) \equiv \boldsymbol{p}_{1}$ (marked $\triangle$ ) on the surface $\Sigma_{1}$. At time $T_{1}=t_{1}$, the trajectory crosses surface $\Sigma_{2}$ at point $\gamma_{1}\left(t_{1}\right) \equiv \boldsymbol{p}_{2} \equiv \gamma_{2}(0)$ (marked $\square$ ). The $j$ th segment of the limit cycle travels from $\gamma_{j}(0) \in \Sigma_{j}$ to $\gamma_{j}\left(t_{j}\right) \in \Sigma_{j+1}$ in time $t_{j}$; the crossing from region $j$ to region $j+1$ occurs at global time $T_{j}=t_{1}+t_{2}+\cdots+t_{j}$ and at location $\gamma_{j}\left(t_{j}\right) \equiv \boldsymbol{p}_{j+1} \equiv \gamma_{j+1}(0)$. The cycle returns to its starting point, $\boldsymbol{p}_{1}$ (marked $\triangle$ ), at time $T_{K}=t_{1}+\cdots+t_{K}$. Thus $T=T_{K}$ is the period of the limit cycle.

$i=1, \ldots, n-1$, and a unique normal vector $\hat{\boldsymbol{n}}_{k+1}$ directed from region $k$ towards region $k+1$. Moreover, the vector fields $\boldsymbol{F}_{k}$ and $\boldsymbol{F}_{k+1}$ are assumed to satisfy a transverse crossing condition:

$$
\begin{array}{r}
\boldsymbol{F}_{k}\left(\boldsymbol{p}_{k+1}\right) \cdot \hat{\boldsymbol{n}}_{k+1}>0 \\
\boldsymbol{F}_{k+1}\left(\boldsymbol{p}_{k+1}\right) \cdot \hat{\boldsymbol{n}}_{k+1}>0 .
\end{array}
$$

(3) The LC of system (2.3) admits a phase function that can be extended to a continuous function $\theta: B . A . \rightarrow S^{1}$ from the basin of attraction to the circle $S^{1} \equiv[0,1]$, satisfying

$$
\frac{d}{d t} \theta(x(t))=\frac{1}{T}
$$

along trajectories within the basin of attraction.

(4) The level sets of the phase function $\theta$ (the isochronal surfaces) form a continuous foliation of an open neighbourhood of the LC.

(5) The phase function, $\theta$, is differentiable within the interior of each region for which it is defined.

(6) At each boundary crossing point $\boldsymbol{p}_{k+1} \in \Sigma_{k+1}$, the directional derivative of the phase function is defined in the directions of each of the $n-1$ tangent vectors $\hat{\boldsymbol{w}}_{i}^{k+1}$.

Remark 2.1 Although we label conditions 1-6 as 'assumptions', it has been recently established that conditions 3-6 follow from conditions 1-2 [56]. All six conditions hold for the model systems we consider in the examples. 
For smooth systems, the iPRC $z(t)=\nabla \theta(\gamma(t))$ may be found using an adjoint equation,

$$
\frac{d z(t)}{d t}=A(t) z(t)
$$

where $A(t)=-D F^{T}(\gamma(t))$, the negative transpose of the linearization of the vector field $\boldsymbol{F}$ evaluated along the LC $\boldsymbol{\gamma}$. To derive (2.6), one considers an infinitesimal perturbation $\boldsymbol{x}(t)=\boldsymbol{\gamma}(t)+\boldsymbol{y}(t)$, where $\|\boldsymbol{y}(t)\| \ll 1$ and $\boldsymbol{\gamma}(t)$ is the LC. As observed in [4], $\boldsymbol{y}(t) \cdot \boldsymbol{z}(t)$ is independent of time; setting

$$
\frac{d}{d t}(\boldsymbol{y}(t) \cdot z(t))=0
$$

gives an operator equation leading to the adjoint. We note that (2.7) holds for piecewise smooth systems within the interior of each subdomain. We will develop a parallel method for piecewise smooth vector fields and solve for each LC section $\gamma_{k}$, that is,

$$
\frac{d z_{k}(t)}{d t}=A_{k}(t) z_{k}(t)
$$

where $A_{k}(t)=-D F_{k}\left(\gamma_{k}(t)\right)^{T}$, the negative transpose of the linearization of the vector field $\boldsymbol{F}_{k}$ evaluated along the LC portion $\gamma_{k}$. The remaining challenge, and the contribution of the paper, is to establish the conditions relating the iPRC on either side of each boundary crossing.

\subsection{Solving the boundary problem of the adjoint equation}

We fix notation and define additional terms. Let $\boldsymbol{F}_{k}$ denote the vector field in which the $k$ th portion of the LC resides, where each $\boldsymbol{F}_{k}$ is numbered sequentially.

The LC, $\gamma$, is piecewise smooth, consisting of several curves $\gamma_{1}, \gamma_{2}, \ldots, \gamma_{K}$. As illustrated in Figure 2, each $\gamma_{k}$ spends a time $t_{k}$ in some domain $D_{r}$. We write the LC $\gamma$ as a collection of curves,

$$
\gamma(t)=\left\{\begin{array}{l}
\gamma_{1}(t), \quad 0=T_{0} \leqslant t<T_{1}, \\
\gamma_{2}\left(t-T_{1}\right), \quad T_{1} \leqslant t<T_{2}, \\
\vdots \\
\gamma_{K}\left(t-T_{K-1}\right), \quad T_{K-1} \leqslant t<T_{K},
\end{array}\right.
$$

where $T_{i}=\sum_{j=1}^{i} t_{j}$ is the global time at which the trajectory crosses boundary surface $\Sigma_{i+1}$, and $\gamma_{k}\left(t_{k}\right)=\gamma_{k+1}(0)$ enforces continuity of the LC. At a LC boundary crossing $\Sigma_{k+1}$ between the $k$ th and $(k+1)$-st portions of the LC, there exist two adjacent vector fields $\boldsymbol{F}_{k}$ and $\boldsymbol{F}_{k+1}$. These vector fields evaluated at the LC boundary crossing are denoted

$$
\begin{aligned}
\boldsymbol{F}_{k, t_{k}} & =\lim _{t \rightarrow t_{k}^{-}} \boldsymbol{F}_{k}\left(\gamma_{k}(t)\right), \\
\boldsymbol{F}_{k+1,0} & =\lim _{t \rightarrow 0^{+}} \boldsymbol{F}_{k+1}\left(\gamma_{k+1}(t)\right) .
\end{aligned}
$$


In equation (2.10) and for the rest of this section, unless stated otherwise, the value $t$ will refer to the time elapsed within a particular region between boundary crossings, i.e. for the $k$ th $L C$ segment, $t \in\left[0, t_{k}\right)$. The one-sided limits exist because each vector field is required to be smooth on the closure of its domain. The global iPRC, $z$, will be defined in terms of the phase variable $\theta \in[0,1)$, but we will view the local iPRC $z_{k}(t)$ in terms of local time. The independent variable of these iPRCs are related by

$$
z(\theta)=z_{k}\left(\theta T-T_{k-1}\right)=z_{k}(t)
$$

We will use the local time $t$ in the proofs to follow, so that we only need to consider local dynamics at an arbitrary boundary crossing, without having to refer to the global dynamics.

We define additional terms $z_{k, t_{k}}$ and $z_{k+1,0}$ by

$$
\begin{gathered}
\boldsymbol{z}_{k, t_{k}}=\lim _{t \rightarrow t_{k}^{-}} z_{k}(t), \\
z_{k+1,0}=\lim _{t \rightarrow 0^{+}} z_{k+1}(t),
\end{gathered}
$$

where $z_{k}$ and $z_{k+1}$ are the solutions to the adjoint equation (equation (2.8)) over vector fields $\boldsymbol{F}_{k}$ and $\boldsymbol{F}_{k+1}$, respectively. As a rule, the first entry of the subscript for either $\boldsymbol{z}_{k, t}$ or $\boldsymbol{F}_{k, t}$ denotes the LC section, and the second entry of the subscript (when explicit) denotes the local time.

Theorem 2.2 Consider a particular domain boundary $\Sigma$ and a piecewise smooth LC $\gamma(t)$ satisfying hypotheses $\mathrm{H} 1-\mathrm{H} 4$ and assumptions 1-6 that transversely crosses $\Sigma$ at point $\mathbf{p}$ at time $t=0$, exiting the old domain with velocity $\boldsymbol{F}^{-}$and entering the new domain with velocity $\boldsymbol{F}^{+}$. For brevity, write $\mathbf{z}^{-}$for the iPRC vector $\lim _{t \rightarrow 0^{-}} \mathbf{z}(t)$ just before the crossing, and $\mathbf{z}^{+}$for the iPRC vector $\lim _{t \rightarrow 0^{+}} \mathbf{z}(t)$ immediately after the crossing. In the interior of domain $k$, the iPRC vector evolves according to $\dot{\mathbf{z}}=-\left(D \mathbf{F}_{k}(\gamma(t))\right)^{\top} \mathbf{z}$. The boundary crossing induces a linear jump condition $C \mathbf{z}^{+}=D \mathbf{z}^{-}$. If $\boldsymbol{w}_{1}, \ldots, \boldsymbol{w}_{n-1}$ is any orthonormal basis for the tangent space of $\Sigma$ at $\boldsymbol{p}$, the matrices $C$ and $D$ are given

$$
C=\left(\boldsymbol{F}^{+}\left|\boldsymbol{w}_{1}\right| \cdots \mid \boldsymbol{w}_{n-1}\right)^{\top}, \quad D=\left(\boldsymbol{F}^{-}\left|\boldsymbol{w}_{1}\right| \cdots \mid \boldsymbol{w}_{n-1}\right)^{\top} .
$$

For convenience, we write

$$
M_{k+1}=C_{k+1}^{-1} D_{k},
$$

and call $M_{k+1}$ the 'jump matrix' for the boundary crossing from region $k$ to $k+1$. Existence of the required matrix inverse is guaranteed by the transverse flow condition.

Proof outline: Requiring that the directional derivatives match at the boundary results in $n-1$ equations with $n$ unknowns. The final equation is determined by matching the normalization condition $F \cdot z=1 / T$ on both sides of the boundary. The resulting system is linear, and can be expressed in the matrix form above. See Section A.1 for the proof of Theorem 2.2. 
The following two corollaries specialize to the case of PWL vector fields. In this case, the vector field is not only piecewise smooth, but the iPRC may be obtained in terms of matrix exponentials.

Corollary 2.3 With the assumptions of Theorem 2.2 and affine linear vector fields $\boldsymbol{F}_{k}$, the initial condition of the iPRC, $z(\theta)$, must satisfy

$$
z_{1,0}=B z_{1,0}
$$

where

$$
B=M_{1} e^{A_{K} t_{K}} M_{K} \cdots M_{k+1} e^{A_{k} t_{k}} M_{k} \cdots M_{3} e^{A_{2} t_{2}} M_{2} e^{A_{1} t_{1}},
$$

$t_{k}$ is the time of flight of the kth portion of the $L C, e^{A_{k} t_{k}}$ is the matrix exponential solution of the adjoint equation with $A_{k}=-\left(D F_{k}\right)^{\top}$ at time $t_{k}$, and $D F_{k}$ denotes the Jacobian matrix of the vector field $\boldsymbol{F}_{k}$. Equation (2.15) and the normalization condition,

$$
\boldsymbol{F}_{1,0} \cdot z_{1,0}=\frac{1}{T}
$$

yield a unique solution for the initial condition, $z_{1,0} \in \mathbb{R}^{n}$.

Proof outline: By iterating the adjoint solution and jump matrices forward in time, we must return to the same initial condition, which results in equation (2.15). This equation is an eigenvalue problem, and reveals that the initial condition of the iPRC is the unit eigenvector of the matrix $B$ up to scalar multiplication. The scalar multiple is uniquely determined by the normalization condition $F \cdot z=1 / T$. See Section A.2 for the proof of Corollary 2.3

Corollary 2.4 Let $t$ denote global time. Under the assumptions of Corollary 2.3, the iPRC is given by

$$
z(t)= \begin{cases}e^{A_{1} t} z_{1,0} \equiv e^{A_{1} t} B z_{1,0} & 0 \leqslant t<T_{1} \\ e^{A_{2}\left(t-T_{1}\right)} B_{1} z_{1,0} & T_{1} \leqslant t<T_{2} \\ \vdots & \\ e^{A_{K}\left(t-T_{K-1}\right)} B_{K-1} z_{1,0} & T_{K-1} \leqslant t<T_{K},\end{cases}
$$

where

$$
\begin{aligned}
B_{1} & =M_{2} e^{A_{1} t_{1}} \\
B_{2} & =M_{3} e^{A_{2} t_{2}} M_{2} e^{A_{1} t_{1}} \\
\vdots & \\
B_{K-1} & =M_{K} e^{A_{K-1} t_{K-1}} \cdots M_{3} e^{A_{2} t_{2}} M_{2} e^{A_{1} t_{1}},
\end{aligned}
$$

and

$$
T_{k}=\sum_{i=1}^{k} t_{i}, \quad k=1, \ldots, K,
$$

where $t_{i}$ denotes the time of flight of the ith portion of the $L C$. 
Proof outline: The iPRC solution is a result of iterating the adjoint equation solution and jump matrices forward in time. See Section A.3 for the proof of Corollary 2.4.

Remark 2.5 For examples of the matrices $M_{k+1}$, see equations (3.2) (Glass network), [46] (Iris system), and (B 6), (B 8), (B 10) (3-D PWL central pattern generator).

Shaw et al. 2012 considered a PWL system satisfying Assumptions 1-6, where the vector field is piecewise differentiable but discontinuous at subdomain boundaries. Our theory correctly captures all discontinuities of the iPRC at the boundaries [55]. Coombes (2008) considered systems with continuous vector fields not necessarily differentiable at domain boundaries, and analytically computed continuous iPRCs for each system. In the following corollary, we show that a continuous iPRC is a general property of LCs over continuous, piecewise smooth vector fields.

Corollary 2.6 Under the assumptions of Corollary 2.3, if adjacent vector fields evaluated along the $L C, \boldsymbol{F}_{k+1,0}$ and $\boldsymbol{F}_{k, t_{k}}$, are continuous at the boundary $\boldsymbol{p}_{k+1}$, then the matrix $M_{k+1}$ is the identity matrix.

Proof outline: If the vector field is continuous, then the solution velocity before the crossing $\left(F^{-}\right)$is equal to the velocity after the crossing $\left(F^{+}\right)$. Since all other terms in equation (4.1) are the same, and the jump matrix is the product of a matrix times its inverse, the jump matrix reduces to the identity. See Section A.4 for the proof of Corollary 2.6.

Remark 2.7 Our analysis therefore includes the iPRC calculations of [8] and [55] as special cases.

\section{Results}

We apply our analysis to several examples in order of increasing complexity. The first is a piecewise constant system with the vector fields arranged such that a LC exists (Section 3.1). The second example (Section 3.2) is a planar system introduced in [55], motivated by investigations of heteroclinic channels as a dynamical architecture for motor control. The third example (Section 3.3) is a 2-D Glass network, a PWL system obtained as the singular limit of a class of models for feedback inhibition and gene regulatory networks [19]. The fourth example (Section 3.4) is a 3-D PWL system arising as a simplification of a nominal central pattern generator model for regulation of feeding motor activity in the marine mollusk Aplysia californica [37,54] and related to a Lotka-Volterra system with three populations [44]. In the final two examples, we extend our theory to the case of weakly coupled oscillators (Section 4.1). In the first weakly coupled example (Section 3.4.1), we take a pair of weakly coupled piecewise constant models from Section 3.1 (for a total of four dimensions) and analyze the existence, stability, and time-course of synchronous and anti-phase states. Finally, in Section 3.4.2, we consider a pair of weakly coupled PWL models (a total of six dimensions), and show that the weak coupling theory accurately predicts the time-course and synchronization properties of the network. 


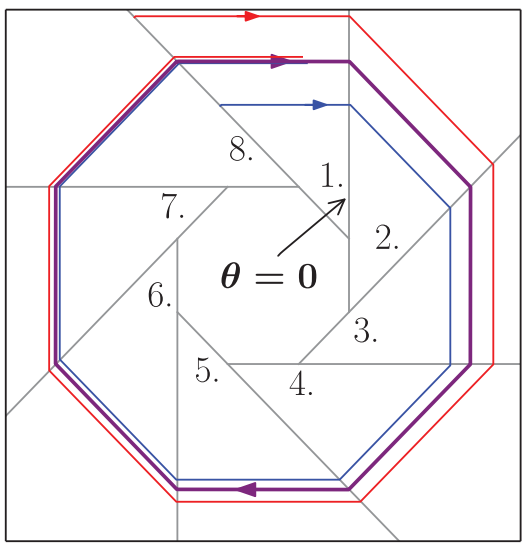

$$
f(\mathbf{x})=16 \begin{cases}\left(\begin{array}{c}
1 \\
0
\end{array}\right), & \mathbf{x} \in\{-y+\sqrt{2} \leq x<1\} \\
\left(\begin{array}{c}
1 / \sqrt{2} \\
-1 / \sqrt{2}
\end{array}\right), & \mathbf{x} \in\{1 \leq x<y+\sqrt{2}\} \\
\left(\begin{array}{c}
0 \\
-1
\end{array}\right), & \mathbf{x} \in\{-1 \leq y<x-\sqrt{2}\} \\
\left(\begin{array}{l}
-1 / \sqrt{2} \\
-1 / \sqrt{2}
\end{array}\right), & \mathbf{x} \in\{-x-\sqrt{2} \leq y<-1\} \\
\left(\begin{array}{c}
-1 \\
0
\end{array}\right), & \mathbf{x} \in\{-1 \leq x<-y-\sqrt{2}\} \\
\left(\begin{array}{c}
-1 / \sqrt{2} \\
1 / \sqrt{2}
\end{array}\right), & \mathbf{x} \in\{y-\sqrt{2} \leq x<-1\} \\
\left(\begin{array}{c}
0 \\
1
\end{array}\right),, & \mathbf{x} \in\{x+\sqrt{2} \leq y<1\} \\
\left(\begin{array}{l}
1 / \sqrt{2} \\
1 / \sqrt{2}
\end{array}\right), & \mathbf{x} \in\{1 \leq y<-x+\sqrt{2}\}\end{cases}
$$

FIGURE 3. The domain and solutions of the piecewise constant system. On the left panel, domains are labelled with numbers $1-8$. We choose zero phase to be the vertical boundary between regions 1 and 2. The limit cycle solution is shown in purple, while example trajectories inside and outside the limit cycle are shown in blue and red, respectively. The right panel specifies the dynamics of each region with the corresponding domain. We define each set such that it implicitly contains all $(x, y) \in \mathbb{R}^{2}$ satisfying the inequalities. For all remaining $\mathbf{x}$ (i.e., within the central octagonal region) we use $d \mathbf{x} / d t=\mathbf{x}$.

\subsection{Piecewise constant model}

To highlight the necessity of our theory, we explore a simple planar example consisting solely of piecewise constant vector fields. Through naïve application of classic theory, one would expect that because the Jacobian matrix of each region is zero (due to the constant nature of each region), that the iPRC would also be constant. However, this is not the case as shown by numerical simulations (Figure 4).

LCs - closed, isolated periodic orbits - do not occur in linear dynamical systems. It is particularly striking, therefore, when they arise in a Filippov system, the right-hand side of which is piecewise constant. In Figure 3, we show the domain of the system with example trajectories that converge to the LC (purple). On the right panel of the same figure, we define the vector field and corresponding domain.

Traditional application of the adjoint method suggests, incorrectly, that the solution to the adjoint equation is constant, because the Jacobian matrix in each region is zero. Standard numerical calculation of the iPRC (dots in Figure 4) shows that the traditional approach is not sufficient. The numerics suggest that the iPRC is constant except on a set of measure zero, where it jumps discontinuously to other values.

As a check of our analytical results, we numerically evaluate the iPRC at a given phase as follows. We integrate the LC up to the given phase, then re-initialize the solution at a new position in the direction of a standard basis vector of magnitude between 1e-2 and 1e-4. This process effectively applies an infinitesimal delta function perturbation in phase space. After integrating for a sufficiently long time (typically 10 times the period), we record the timing difference between the unperturbed and perturbed LCs. We then divide this value by the magnitude of the perturbation. This magnitude is sufficiently small to give an accurate approximation to the true $\mathrm{PRC}$ for the systems here. 


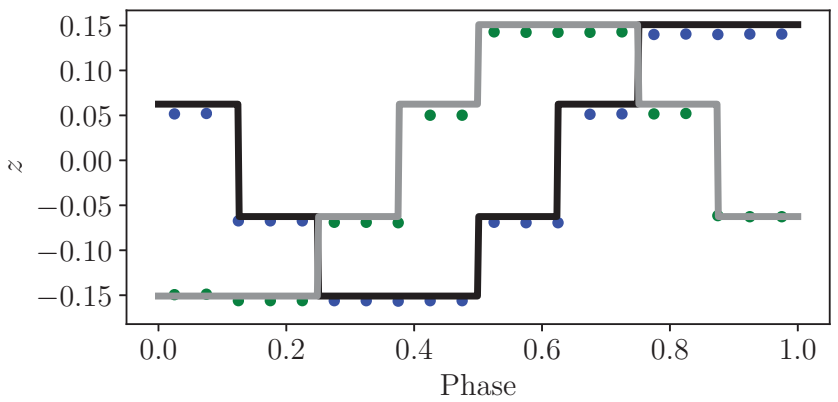

FigURE 4. iPRC of the piecewise constant model. The numerically computed iPRC is shown in blue (green) for the first (second) coordinate. The first (second) coordinate of the analytically computed iPRC function is shown in black (gray). The piecewise constant vector field leads to a piecewise constant iPRC. The components take the values $\pm \frac{1}{16}= \pm 0.0625$ and $\pm 1 /(16(\sqrt{2}-1)) \approx \pm 0.1509$.

Using Theorem 2.2, we compute the size of the discontinuities exactly. The iPRC of both coordinates are shown in Figure 4. Note that the Jacobian is identically zero within each domain, so the stability of the LC arises from the contraction of adjacent trajectories (by a factor of $1 / \sqrt{2}$ ) at each boundary crossing.

This example highlights the necessity of our theorem to compute the iPRC for oscillators in piecewise smooth systems. However, application of Theorem 2.2 extends well beyond this simple, constructed example. In the examples to follow, we show the broad applicability of our theorem by analyzing several existing models in literature. We begin with a symmetric PWL model from [55].

\subsection{Piecewise linear Iris system with non-uniform saddle values}

In this section, we briefly discuss a planar model for which the iPRC is explicitly computable [55], and how our theory extends this calculation.

As part of [55], we analyzed a PWL system (the iris system), where a stable heteroclinic cycle gives rise to a one-parameter family of LCs similar to those in the sine system [55]. The PWL analogue consists of four regions with velocity fields equivalent, under successive 90-degree rotations, to the velocity field in the unit square. Our theory reproduces the result immediately as shown in [46]. Moreover, while the result in [55] depends on symmetry of the system, Theorem 2.2 naturally accounts for the same system with heterogeneous vector fields,

$$
\begin{aligned}
\frac{d \hat{s}_{k}}{d t} & =-\lambda_{k} \hat{s}_{k}, \\
\frac{d \hat{u}_{k}}{d t} & =\hat{u}_{k},
\end{aligned}
$$

where $\lambda_{k}$ for $k \in\{1,2,3,4\}$. Despite the loss of symmetry, the calculations in this case follow the same steps as in [46]. Next, we turn to a classic, biologically motivated model with asymmetric PWL vector field dynamics. 


\section{(a)}

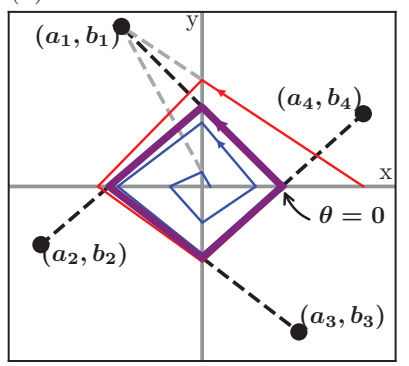

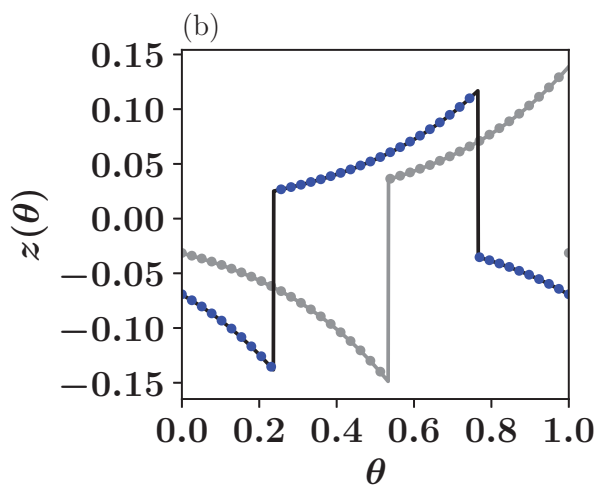

FIGURE 5. (a): A model of feedback inhibition as discussed in Example 1 of Glass and Pasternack 1978. The limit cycle attractor (purple) traverses four quadrants, which serve as the four domains of the model. We call the first quadrant region 1, and because the solutions travel counter-clockwise, the second quadrant is named region 2, the third quadrant region 3, and the fourth quadrant region 4. Within each quadrant, trajectories are attracted to a target point outside the domain, as shown by the black and gray dashed lines. For example, in region 1 , the limit cycle trajectory (purple) is attracted to the target point $\left(a_{1}, b_{1}\right)$ until it hits the positive $y$-axis, at which point the limit cycle trajectory changes direction towards the next target point, $\left(a_{2}, b_{2}\right)$. Two sample trajectories in the same region, one inside (blue) and one outside (red) the limit cycle, demonstrate that the purple loop is indeed a limit cycle attractor. The target points are $\left(a_{1}, b_{1}\right)=(-5,11),\left(a_{2}, b_{2}\right)=(-10,-4)$, $\left(a_{3}, b_{3}\right)=(6,-10)$, and $\left(a_{4}, b_{4}\right)=(10,5)$. (b): The numerical (dots) and analytical (lines) infinitesimal phase response curves of the planar Glass network model. The analytical solution to the adjoint equation is given by equation (2.19); the numerical iPRC is calculated via direct perturbation. Blue curve: iPRC for perturbations along the horizontal axis. Gray curve: iPRC for perturbations along the vertical axis.

\subsection{Two dimensional Glass network}

Glass, Perez, and Pasternack introduced a planar PWL system as a model of feedback inhibition in a genetic regulatory circuit $[19,20]$. Figure 5(a) illustrates several trajectories converging to a stable LC in such a network. The concentration $x_{1}$ stimulates the production of $x_{2}$, while $x_{2}$ inhibits the production of $x_{1}$. One may also consider a macroscopic analogue in a predator-prey system, where $x_{1}$ is the prey and $x_{2}$ is the predator. Within each quadrant of the plane, the trajectories converge towards a target point located in the subsequent quadrant (Figure 5(a)).

The LC attractor, its time of flight through each region, and its points of intersection with each axis is found by using Poincare maps. We refer the reader to the references above for the details. By Theorem 2.2, the jump matrices are

$$
\begin{array}{ll}
M_{1}=\left(\begin{array}{cc}
1 & 0 \\
\frac{a_{4}-a_{1}}{b_{1}} & \frac{b_{4}}{b_{1}}
\end{array}\right), & M_{2}=\left(\begin{array}{cc}
\frac{a_{1}}{a_{2}} & \frac{b_{1}-b_{2}}{a_{2}} \\
1 & 0
\end{array}\right), \\
M_{3}=\left(\begin{array}{cc}
1 & 0 \\
\frac{a_{2}-a_{3}}{b_{3}} & \frac{b_{2}}{b_{3}}
\end{array}\right), \quad M_{4}=\left(\begin{array}{cc}
\frac{a_{3}}{a_{4}} & \frac{b_{3}-b_{4}}{a_{4}} \\
1 & 0
\end{array}\right) .
\end{array}
$$


By Corollary 2.3, we find that the eigenvector associated with the unit eigenvalue is

$$
z_{1,0}=\frac{\hat{z}_{1,0}}{T\left[b_{1}-\frac{a_{1}}{b_{1}}\left(a_{1}-\left[\boldsymbol{p}_{1}\right]_{1}\right)\right]} .
$$

We now have enough information to generate the iPRC using equation (2.19), which we show in Figure 5(b).

Figure 5(b) shows the iPRC obtained analytically together with the iPRC obtained by direct numerical perturbations. The iPRC components show the sign and size of the effect that a small displacement away from the LC trajectory has on the subsequent timing of the trajectory as it returns to the LC. For example, the descending blue curve (horizontal component) reaches a minimum at the point of egress from the northeastern sector, with a discontinuity occurring where the LC trajectory crosses the ray $x=0, y>0$ (cf. Figure $5(b))$. The timing of the LC has its greatest sensitivity to small perturbations in the $(1,0)$ direction at this point in the cycle; a perturbation in this direction causes a delay in the trajectory upon return to the LC. Similarly, the greatest sensitivity to perturbations in the horizontal direction occur immediately before crossing the ray $y=0, x<0$; a perturbation in the direction $(0,1)$ at this point causes a significant delay. In contrast, the greatest phase advance in response to a horizontal (resp., vertical) perturbation occurs just before the border crossing at approximately $3 / 4$ of the period (resp., 1 full period).

So far our examples have been scalar or planar, but Theorem 2.2 applies to systems in arbitrary dimensions. In our next example, we consider a novel PWL system in three dimensions.

\subsection{Nominal biting model of Aplysia Californica}

In this section, we consider a PWL analogue of the three-dimensional system considered in [54]. Written in the order of regions 1, 2, and 3, respectively, we consider the following system:

$$
\frac{d \boldsymbol{r}}{d t}=\left\{\begin{array}{c}
1-x-(y+a) \rho \\
y+a, \quad x \geqslant y+a, x \geqslant z-a, \\
(z-a)(1-\rho) \\
(x-a)(1-\rho) \\
1-y-(z+a) \rho, \quad y>x-a, y \geqslant z+a, \\
z+a \\
x+a \\
(y-a)(1-\rho), \quad z>x+a, z>y-a, \\
1-z-(x+a) \rho
\end{array}\right.
$$

where $\boldsymbol{r}=(x, y, z), a \geqslant 0$ is the bifurcation parameter, and $\rho$ is the coupling strength. The domains of equation (3.4) lie in equal thirds of the unit cube, which, when $a=0$, all share an edge along the vector $(1,1,1)$. For $a=0$, the domain of region 1 is the convex hull of the vertices $(1,0,0),(1,0,1),(1,1,0)$ and $(1,1,1)$. Similarly, the domain of region 2 is the 

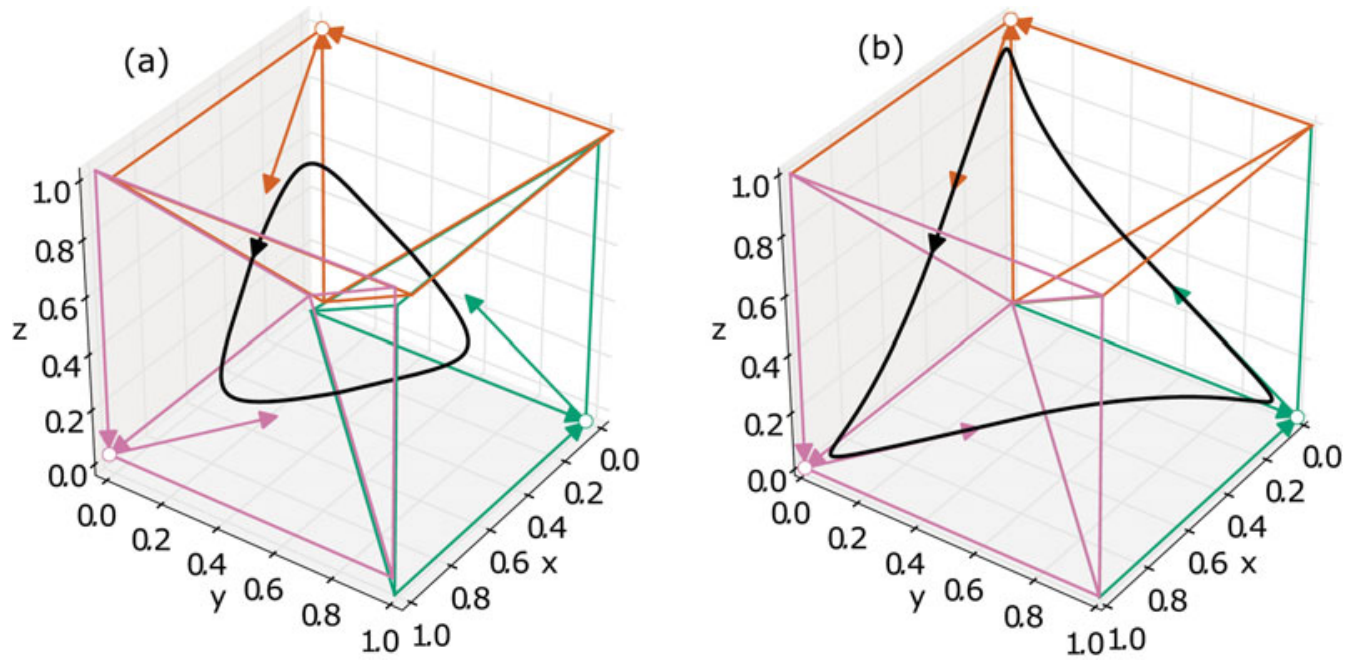

Figure 6. Piecewise linear model of Aplysia motor system for two bifurcation parameter values, $\rho=3, a=0.02$ (a) and $a=0.001$ (b) The three domain boundaries are defined according to equation (3.4), with $\|(x, y, z)\|_{1} \leqslant 1$ when $a=0$. Regions 1,2 and 3 are colored magenta, green and orange, respectively (color online). Each region includes one saddle point, denoted by a circle. The two arrows pointing into the saddle point indicate the two eigenvectors generating the stable manifold, and the arrow pointing away from the saddle indicates the eigenvector generating the unstable manifold (equations (B 1)-(B 3)). The black loop in both figures represents the stable limit cycle, with the black arrow denoting the direction of flow. The tip of the black arrow marks the point $\boldsymbol{p}_{1}$ at the boundary between regions 3 and 1 . We define the phase at this point to be zero.

convex hull of the vertices $(0,1,0),(1,1,0),(0,1,1)$ and $(1,1,1)$, and the domain of region 3 is defined by the vertices $(0,0,1),(1,0,1),(0,1,1)$ and $(1,1,1)$. The saddle points of the system lie on a vertex of each domain, namely at $(1,0,0),(0,1,0)$ and $(0,0,1)$, for regions 1,2 and 3 , respectively.

The calculation of the jump matrices is more involved than the previous examples, so we include additional details in Appendix B and move ahead to the calculation of the initial value of the iPRC.

We continue with $\rho=3$ and $a=0.01$, which is sufficient for a LC solution to exist (Figure 6). By Corollary 2.3, $B$ is given by

$$
B \approx 10 \times\left(\begin{array}{ccc}
1.25 \times 10^{3} & 2.71 & -4.23 \times 10^{2} \\
2.09 \times 10^{4} & 3.18 \times 10 & -2.5 \times 10^{3} \\
1.52 \times 10^{3} & 2.90 & -3.85 \times 10^{2}
\end{array}\right)
$$

with a near-unit eigenvalue of approximately 0.998 . The associated eigenvector, $\hat{z}_{1,0}$ is,

$$
\hat{z}_{1,0} \approx\left(1.15 \times 10^{-3},-1,-2.98 \times 10^{-3}\right)
$$




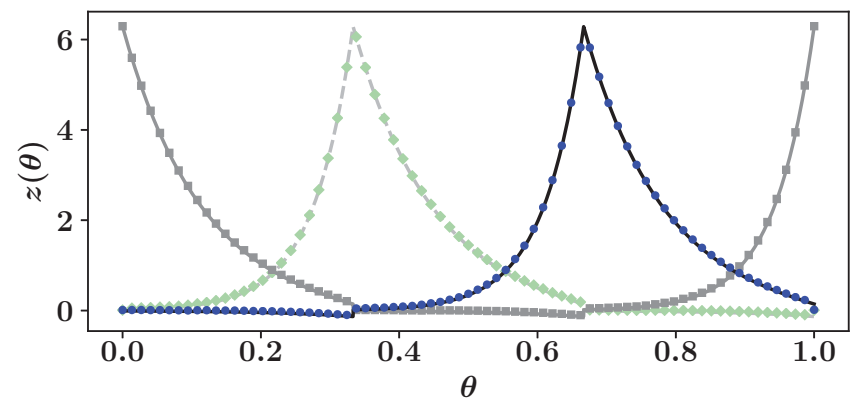

Figure 7. Aplysia motor control model iPRC, for parameters $\rho=3$ and $a=0.01$. The blue dots, gray squares and light green diamonds represent the numerical iPRC, found by the direct method of perturbations, of the first, second, and third components of the iPRC, respectively. The solid black, solid gray, and dashed light gray lines represent the analytical iPRC derived using Theorem 2.2 and Corollary 2.3 of the first, second, and third components of the iPRC, respectively.

As in the preceding examples, the initial condition of the iPRC, $z_{1,0}$, comes from scaling this eigenvector of matrix $B, \hat{z}_{1,0}$, by equation (2.17) of Corollary 2.3 :

$$
\boldsymbol{z}_{1,0}=\frac{\hat{\boldsymbol{z}}_{1,0}}{\left(\hat{\mathbf{z}}_{1,0} \cdot \boldsymbol{p}_{1}\right) T}
$$

The values $\boldsymbol{p}_{1}$ and $T$ represent the initial condition of the LC and the total period of the $\mathrm{LC}$, respectively, and are found numerically.

For the numerical iPRC, we choose the perturbation magnitude to be $10^{-4}$, with width equal to the time step and follow the same procedure used to generate Figure 5(b). Figure 7 plots the resulting analytic iPRC together with the iPRC obtained numerically by the direct perturbation method, showing good agreement. The greatest phase advance in response to a positive perturbation in the $(1,0,0)$ direction (black), $(0,1,0)$ direction (solid gray), and $(0,0,1)$ direction (light dashed gray lines) occurs at phase $2 / 3$ (entry to the region including the point $(0,0,1)$ ), phase 0 (entry to the solid region including the point $(1,0,0)$ ), and phase $1 / 3$ (entry to the region including point $(0,1,0)$ ), respectively.

Each of the preceding examples is consistent with our theory. We now turn to more practical applications. In the final pair of examples to follow, we demonstrate the utility of Theorem 2.2 and iPRCs in general by analyzing weakly coupled piecewise smooth oscillators in 4 and 6 dimensions, respectively.

\subsection{Synchronization of piecewise smooth oscillators}

In the classic theory for smooth systems, synchronization properties of weakly coupled identical oscillators of the form

$$
\begin{aligned}
& \frac{d \mathbf{x}}{d t}=f(\mathbf{x})+\varepsilon G(\mathbf{x}, \mathbf{y}) \\
& \frac{d \mathbf{y}}{d t}=f(\mathbf{y})+\varepsilon G(\mathbf{y}, \mathbf{x}),
\end{aligned}
$$


where $|\epsilon| \ll 1$, may be understood by studying the so-called interaction function, $H$, which is the convolution of the coupling function $G$ with the iPRC of the $T$-periodic LC oscillator $U$ satisfying $\dot{U}=f(U)$. Thus,

$$
H(\phi)=\frac{1}{T} \int_{0}^{T} z(t) \cdot G[U(t), U(t+\phi)] d t,
$$

where $U$ is the LC oscillator, and $z$ is the associated (vector) iPRC [14]. Although the theory assumes that $U$ satisfies both equations (3.8) and (3.9) for $\varepsilon$ small, the identical oscillators may have different phases, $\theta_{1}$ and $\theta_{2}$, respectively. To study synchronization, we consider the phase difference $\psi=\theta_{2}-\theta_{1}$, i.e. we must integrate the scalar differential equation

$$
\frac{d \psi}{d t}=\varepsilon[H(-\psi)-H(\psi)]
$$

Stable fixed points of this equation predict the existence and type of stable phase-locked solutions under weak coupling.

Remark 3.1 If $z(t)$ were constant, then $H(\phi)$ would be constant, and $d \psi / d t$ would be identically zero.

We will refer back to this elementary observation in Section 3.5.1.

As established above, piecewise smooth oscillators may have iPRCs with discontinuities at domain boundaries. By obtaining the form of such discontinuities, our theory allows us to extend weakly coupled oscillator analysis to weakly coupled piecewise smooth oscillators. We present two examples to illustrate this application of the theory: first, we consider coupled LC oscillators in a recently introduced threshold linear network framework [42], and show that our analysis correctly captures not only the correct stable phase-locked state, but also the time course of synchronization. Even more strikingly, we demonstrate synchronization of coupled LC oscillators in a piecewise constant dynamical system. For a piecewise constant system, the Jacobian matrix is identically zero in the interior of each domain. Hence all nonlinear effects - including the existence of LCs and their synchronization properties - may be said to arise entirely through the boundarycrossing behavior analyzed in this paper.

For each example below, we show that our calculations correctly predict not only the type of synchronization, but also the time course of synchronization, demonstrating that we correctly capture the nonlinear dynamics of synchronization in these weakly coupled piecewise smooth LC systems. We begin by coupling a pair of one of our first examples, the piecewise constant system (Figure 3).

\subsubsection{Synchronization of weakly coupling of oscillators with piecewise-constant velocity}

As seen in Section 3.1, the LC of the piecewise constant system arises as a result of the boundaries. Similarly, the iPRC is entirely determined by the boundary effects, and is piecewise constant in the domain interiors. Synchronization effects arise therefore due to the nonlinear effects at the boundaries and can be analyzed using Theorem 2.2. 
We study the synchronization of two such systems under diffusive coupling of the form $\dot{\mathbf{x}}=f(\mathbf{x})+\varepsilon G(\mathbf{x}, \mathbf{y}), \quad \dot{\mathbf{y}}=f(\mathbf{y})+\varepsilon G(\mathbf{y}, \mathbf{x})$, where $\mathbf{x}=\left(x_{1}, x_{2}\right)^{T}, \mathbf{y}=\left(y_{1}, y_{2}\right)^{T}$, and

$$
G(\mathbf{x}, \mathbf{y})=g\left(\begin{array}{l}
\left(y_{1}-x_{1}\right) \\
\left(y_{2}-x_{2}\right)
\end{array}\right) .
$$

Using Corollary 2.3, we find the discontinuities at the boundaries, and analytically derive the iPRC (Figure 4). The matrix $B$ is

$$
B=\left(\begin{array}{cc}
1 & 0 \\
15(1+\sqrt{2}) & 16
\end{array}\right),
$$

which has eigenvalues and associated eigenvectors

$$
\begin{array}{ll}
\lambda_{1}=1 & \lambda_{2}=16 \\
\vec{v}_{1}=\left(\begin{array}{c}
1-\sqrt{2} \\
1
\end{array}\right) \vec{v}_{2}=\left(\begin{array}{l}
0 \\
1
\end{array}\right) .
\end{array}
$$

Following the calculations in Section 3.5, we see that the convolution of a piecewise constant iPRC with a linear (diffusive) coupling function gives a $C^{1}$, piecewise quadratic interaction function (equation (3.10), top right panel of Figure 9). The bottom right panel of Figure 9 shows the right hand side of equation (3.11) for this example. Figure 8 compares the phase difference predicted by the theory (equation (3.11)) to simulations, showing excellent agreement. The first two panels show the state variables near the beginning and end of the simulation, respectively. The LCs (the first LC is shown in shades of orange and the second in shades of dashed blue) begin close to anti-phase, then converge to an in-phase solution. The rightmost panel shows the predicted and simulated phase difference of the oscillators over time. The horizontal dashed line shows the 'prediction' one would obtain if one neglected the effects due to crossing the switching manifolds, namely the absence of synchronization (i.e., $d \psi / d t=0$, see Remark 3.1). Hence, synchronization arises solely from the effects of the switching boundaries.

\subsubsection{Synchronization of weakly coupled oscillators with piecewise-linear velocity}

In [42] Morrison, Curto and colleagues demonstrate that a simple class of competitive threshold-linear networks can exhibit a rich array of nonlinear dynamical behaviors, including stable LCs, quasi-periodic trajectories and chaotic dynamics, as well as coexistence of multiple attractor types within networks of modest dimension. In this section, we study synchronization properties of two weakly coupled LC oscillators within the Morrison-Curto network:

$$
\begin{aligned}
& \frac{d x_{i}}{d t}=-x_{i}+\left[\sum_{j=1}^{3} W_{i j} x_{j}+\alpha(-1-\delta) \sum_{j=1}^{3} y_{j}+\theta\right]_{+}, \\
& \frac{d y_{i}}{d t}=-y_{i}+\left[\alpha(-1-\delta) \sum_{j=1}^{3} x_{j}+\sum_{j=1}^{3} W_{i j} x y_{j}+\theta\right]_{+},
\end{aligned}
$$



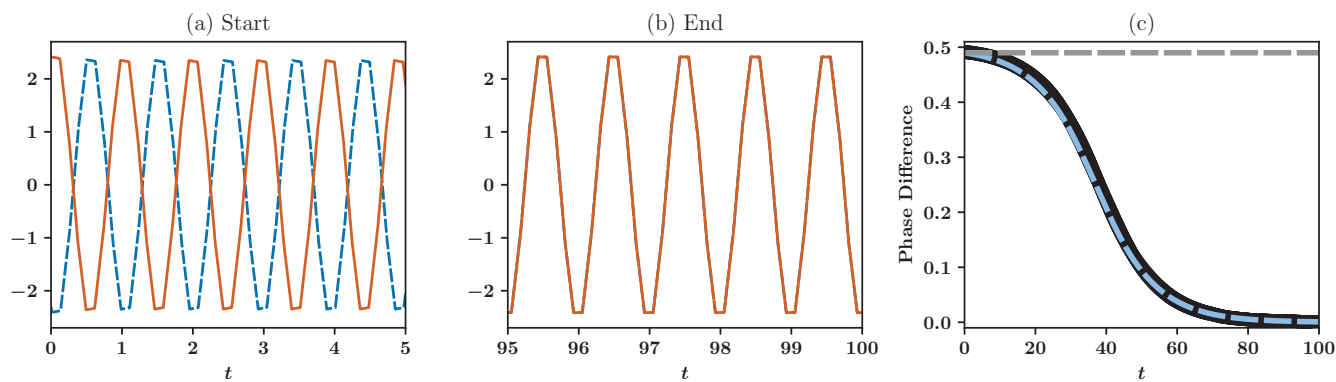

FIGURE 8 . Phase difference of weakly coupled piecewise constant oscillators over time. The first state variable of the first oscillator $\left(x_{1}\right)$ is orange. The first state variable of the second oscillator $\left(y_{2}\right)$ is shown using a dashed blue line. (a) Solutions near the start of the simulation. The dashed blue line is nearly anti-phase to the orange line. (b) Over time, the solutions converge to a synchronous solution. (c) Our predicted phase difference in light blue dashed matches the numerically computed phase difference in black.

(a) Piecewise Constant

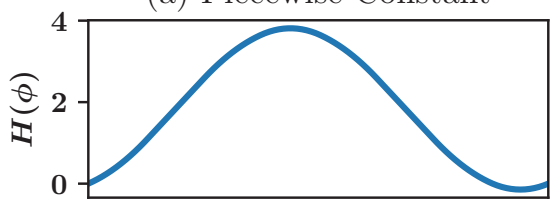

(c)

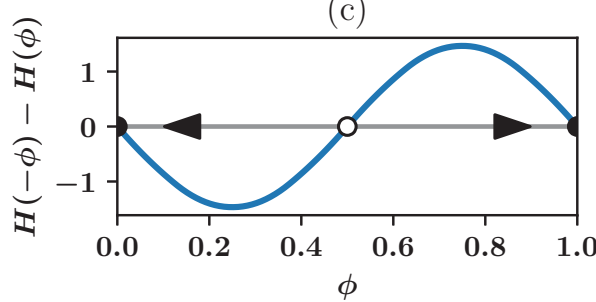

(b) Morrison-Curto

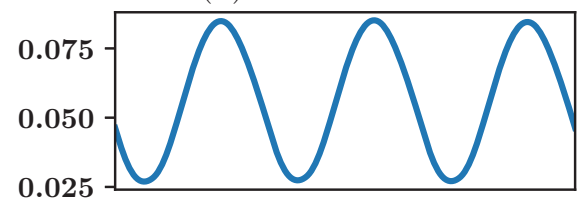

(d)

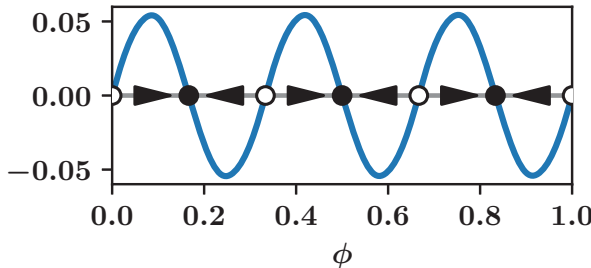

Figure 9. The $H$ functions of the weakly coupled examples. (a): The $H$ function of the piecewise constant system with reciprocal diffusive coupling. (c): The right-hand side of the phase dynamics of the piecewise constant system. All non-fixed point solutions tend towards synchrony. (b): The $H$ function of the Morrison-Curto model. (d): The right-hand side of the phase dynamics of the Morrison-Curto model. The phase dynamics converge to different phase-locked values depending on the initial conditions.

with threshold parameter $\theta=1$,

$$
W=\left(\begin{array}{ccc}
0 & -1-\delta & -1+\varepsilon \\
-1+\varepsilon & 0 & -1-\delta \\
-1-\delta & -1+\varepsilon & 0
\end{array}\right)
$$

and $0 \leqslant \alpha<1$. The threshold nonlinearity $[\cdot]_{+}$is given by $[y]_{+}=\max \{y, 0\}$. With this choice of weight matrix, and for $\alpha=0$, the system exhibits a LC $\mathbf{x}_{0}(t)$ [42]. For sufficiently small $\alpha>0$ the LC persists, with negligible changes in its shape and timing properties as $\alpha$ increases. 
When $\alpha \ll 1$, the coupling term $\alpha(-1-\delta) \sum_{j=1}^{3} y_{j}$ has little effect on the boundary crossing points of the LC, but it does contribute to the dynamics of $x_{i}$, provided the term $\sum_{j=1}^{3} W_{i j} x_{j}$ is above threshold. Thus, to first order in $\alpha$, we may approximate the dynamics as

$$
\frac{d x_{i}}{d t}=-x_{i}+\left[\sum_{j=1}^{3} W_{i j} x_{j}+\theta\right]_{+}+\alpha(-1-\delta) \Theta\left(\sum_{j=1}^{3} W_{i j} x_{j}+\theta\right) \sum_{j=1}^{3} y_{j}
$$

where $\Theta$ is the Heaviside function. In this form, we have a system of weakly coupled oscillators, $d \mathbf{x} / d t=F(\mathbf{x})+\alpha G(\mathbf{x}, \mathbf{y}), d \mathbf{y} / d t=F(\mathbf{y})+\alpha G(\mathbf{y}, \mathbf{x})$, where

$$
F(\mathbf{x})=\left(\begin{array}{ll}
-x_{1}+\left[\sum_{j=1}^{3} W_{1 j} x_{j}+\theta\right. & \\
-x_{2}+\left[\sum_{j=1}^{3} W_{2 j} x_{j}+\theta\right. & ]_{+} \\
-x_{3}+\left[\sum_{j=1}^{3} W_{3 j} x_{j}+\theta\right]_{+}
\end{array}\right),
$$

and

$$
G(\mathbf{x}, \mathbf{y})=\left(\begin{array}{c}
(-1-\delta) \Theta\left(\sum_{j=1}^{3} W_{1 j} x_{j}+\theta\right) \sum_{j=1}^{3} y_{j} \\
(-1-\delta) \Theta\left(\sum_{j=1}^{3} W_{2 j} x_{j}+\theta\right) \sum_{j=1}^{3} y_{j} \\
(-1-\delta) \Theta\left(\sum_{j=1}^{3} W_{3 j} x_{j}+\theta\right) \sum_{j=1}^{3} y_{j}
\end{array}\right)
$$

In this case, although the vector field is not continuously differentiable at the domain boundaries, it is nevertheless continuous, so by Corollary 2.6, we find that the jump matrices are equal to the identity. Thus, we can establish that for this system the iPRC may be obtained by integrating the adjoint equation in the usual fashion ( $c f$. Section 3.5.1 for an example with nontrivial jump matrices). With the iPRC established, we may numerically integrate equations (3.10) and (3.11) to predict the synchronization dynamics of the coupled oscillators. The left column panels of Figure 9 shows the numerically computed $H$ function and right-hand side of the phase difference equation. Figure 10 compares the time course of the phase difference $\psi$ predicted by integrating the $1 \mathrm{D}$ phase equation (3.11) and the phase difference time course obtained by integrating the full 6-D equations (equation (3.15)). The theory and simulations show excellent agreement.

\subsubsection{Numerics and phase estimation}

To estimate the phase of an oscillator in the piecewise constant system, we use the geometrical phase angle of the state variables to approximate the phase of the full model. This approximation is reasonable because the system has a high degree of symmetry, and numerical tests reveal little error between this method and the more involved but general method below.

In the Morrison and Curto model (Section 3.5.2), the system lacks the type of symmetry present in the piecewise constant system. Thus, we resort to a brute force lookup table method used in [47] to estimate the phase. 

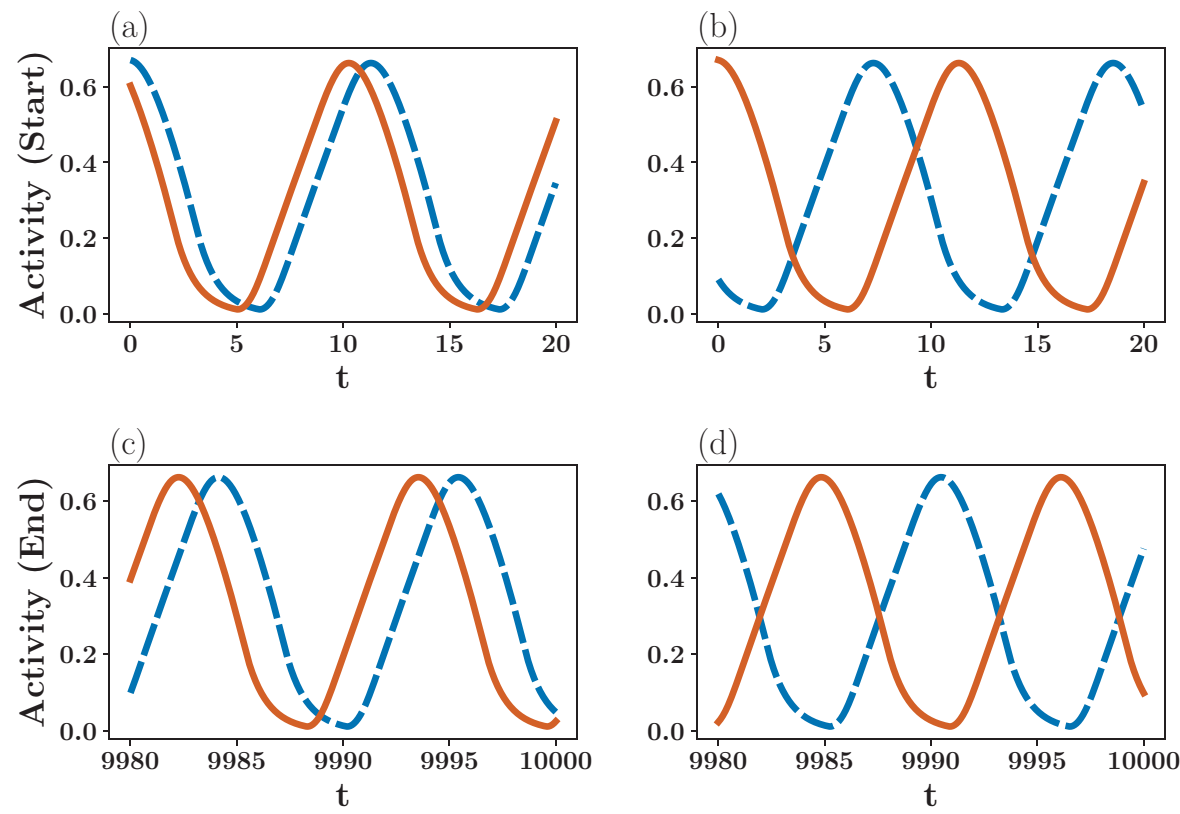

(e)

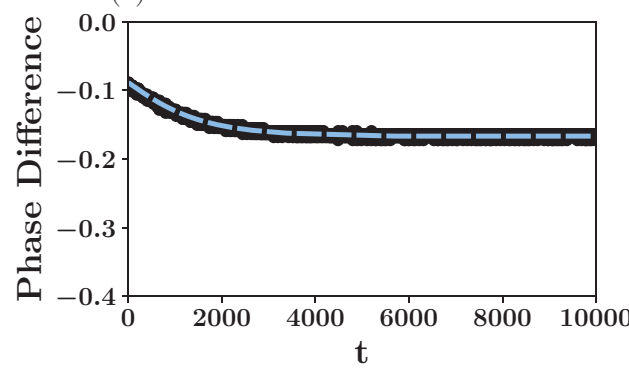

(f)

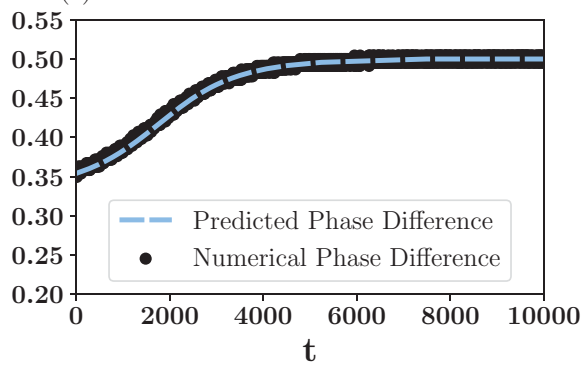

FiguRE 10. Evolution of phase difference for weakly coupled Morrison-Curto competitive threshold-linear network oscillators. The first state variables of each oscillator are shown in orange and blue dashed (corresponding to $x_{1}$ and $y_{1}$, respectively). Left column ((a),(c),(e)): nearsynchronous initial conditions (a) separate slightly to a state with greater phase lag (c) with a phase difference of $-1 / 6$ (e). Right column ((b), (d), (f)): near-antisynchronous initial conditions (b) converge to an anti-phase solution (d), with a phase difference of $1 / 2$. Bottom row ((e), (f)): time course of phase difference predicted from reduced phase description, equation (3.11) (light blue dashed line), matches that of full 6D system (black solid line). Parameters $\alpha=0.01, \delta=0.5, \varepsilon=0.25$. See also Section 3.5.3.

For all simulations of the Morrison-Curto model, we use the parameter values $\delta=.5$, $\varepsilon=.25, \theta=1$, and $\alpha=.01$. To reduce the number of redundant plot points, we plot every 500 points.

All figure code, including data files and files for data generation is available on GitHub at https://github.com/youngmp/pwl_iprc. 


\section{Discussion}

\subsection{Relation to the saltation matrix}

In this paper, we have derived the form of the discontinuity in the iPRC at domain boundaries for a generic LC arising in a piecewise smooth dynamical system in arbitrary finite dimensions. The solution of this problem is closely related to the solution of the variational problem for piecewise smooth systems, as we now discuss.

First, to recapitulate our result, consider a trajectory $\gamma(t)$ transversely crossing domain boundary $\Sigma$ at point $\mathbf{p}$ at time $t=0$, exiting the old domain with velocity $\boldsymbol{F}^{-}$and entering the new domain with velocity $\boldsymbol{F}^{+}$. For brevity, write $\mathbf{z}^{-}$for the iPRC vector $\lim _{t \rightarrow 0^{-}} \mathbf{z}(t)$ just before the crossing, and $\mathbf{z}^{+}$for the iPRC vector $\lim _{t \rightarrow 0^{+}} \mathbf{z}(t)$ immediately after the crossing. In the interior of domain $k$, the iPRC vector evolves according to $\dot{\mathbf{z}}=-\left(D \mathbf{F}_{k}(\gamma(t))\right)^{\top} \mathbf{z}$. The boundary crossing induces a linear jump condition $C \mathbf{z}^{+}=D \mathbf{z}^{-}$. If $\boldsymbol{w}_{1}, \ldots, \boldsymbol{w}_{n-1}$ is any orthonormal basis for the tangent space of $\Sigma$ at $\boldsymbol{p}$, the matrices $C$ and $D$ are given

$$
C=\left(\boldsymbol{F}^{+}\left|\boldsymbol{w}_{1}\right| \cdots \mid \boldsymbol{w}_{n-1}\right)^{\top}, \quad D=\left(\boldsymbol{F}^{-}\left|\boldsymbol{w}_{1}\right| \cdots \mid \boldsymbol{w}_{n-1}\right)^{\top},
$$

$c f$. equation (2.14).

A linear jump condition arises as well in the solution of the variational problem for piecewise smooth systems. The solution was obtained by Aizerman and Grantmakher [2] and is discussed in the monographs [11,32]. Within the interior of the $k$ th domain, the evolution of a small perturbation $\boldsymbol{u}(t)$ to a trajectory $\boldsymbol{y}(t) \approx \gamma(t)+\boldsymbol{u}(t)$ evolves, to linear order, as $\dot{\boldsymbol{u}}=D \boldsymbol{F}_{k}(\gamma(t)) \boldsymbol{u}$. Writing $\boldsymbol{u}^{-}=\lim _{t \rightarrow 0^{-}} \boldsymbol{u}(t)$ and $\boldsymbol{u}^{+}=\lim _{t \rightarrow 0^{+}} \boldsymbol{u}(t)$, the jump in $\boldsymbol{u}$, upon $\gamma$ crossing the boundary $\Sigma$ at $\boldsymbol{p}$, is given by the Saltation matrix $S$. That is, $\boldsymbol{u}^{+}=S \boldsymbol{u}^{-}$, where the matrix $S=I+\Delta \boldsymbol{F} \hat{\boldsymbol{n}}^{\top} /\left(\hat{\boldsymbol{n}} \cdot \boldsymbol{F}^{-}\right)$is defined in terms of the difference in the vector fields, $\Delta \boldsymbol{F} \equiv \boldsymbol{F}^{+}-\boldsymbol{F}^{-}$, and the vector $\hat{\boldsymbol{n}}$ normal to the surface $\Sigma$ at $\boldsymbol{p}$.

Because the basis vectors satisfy $\boldsymbol{w}_{i} \perp \hat{\boldsymbol{n}}$ for each $i$, the matrices $A$ and $B$ satisfy the equations

$$
(S-I) B^{\top}=(\Delta \boldsymbol{F}|\mathbf{0}| \cdots \mid \mathbf{0})=-\left(S^{-1}-I\right) A^{\top} .
$$

Comparing (4.1)-(4.2), notice that $C^{\top}-D^{\top}=(S-I) D^{\top}$. It follows immediately that the jump matrix can be written in terms of the saltation matrix:

$$
M=C^{-1} D=\left(S^{-1}\right)^{\top} .
$$

This relation is quite natural: The saltation matrix accounts for changes in the fate of trajectories with perturbed initial conditions with respect to evolution forward in time; it corrects the linearized forward (variational) equation. The jump matrix obtained in this paper accounts for changes when tracking the prior history of trajectories that would have converged to common points on a LC; it corrects the linearized backward equation for the effects of boundary crossing.

Alternatively, consider the following elementary derivation.

Remark 4.1 Assume the isochrons are given by a piecewise smooth function $\theta(\mathbf{u})$. The phase difference between any two points remains constant in time as the trajectories through those 
two points evolve. Thus, for any two points $\mathbf{x}$ and $\mathbf{x}+\mathbf{u}$,

$$
\frac{d}{d t}(\theta(\mathbf{x}+\mathbf{u})-\theta(\mathbf{x}))=0 .
$$

Introducing the gradient $\mathbf{z}(t) \equiv \nabla \theta(\gamma(t))$ and considering an arbitrary small displacement $\mathbf{u}$, we see that

$$
\mathbf{z} \cdot \mathbf{u}=\text { const }
$$

along trajectories, both within the interior of a region, and across the switching boundaries. When $\mathbf{u}$ jumps, in general so does $\mathbf{z}$. We seek a matrix $M$ satisfying $\mathbf{z}_{+}=M \mathbf{z}_{-}$, where $\mathbf{z}_{-}$is the iPRC just before the switching boundary and $\mathbf{z}_{+}$is the iPRC just after. Preserving $\mathbf{z} \cdot \mathbf{u}$ requires

$$
\mathbf{z}_{-}^{\top} \mathbf{u}_{-}=\mathbf{z}_{+}^{\top} \mathbf{u}_{+}=\mathbf{z}_{-}^{\top} M^{\top} S \mathbf{u}_{-}
$$

for arbitrary small perturbations $\mathbf{u}$. Therefore, $M^{\top} S=I$, or $M=\left(S^{\top}\right)^{-1}$, as shown above.

\subsection{Related literature}

Saltation matrices, or closely related constructions, appear in the analysis of iPRCs in a handful of papers that we now review.

We first derived the general form of the jump condition for the iPRC of a continuous LC trajectory in arbitrary dimensions in [46] without reference to saltation matrix methods. Subsequently, [56] used the saltation matrix to account for jumps in the iPRC for general hybrid systems, allowing for discontinuous solutions. In fact, our result is similar and complementary to [56]. In [56] and the present study, we require differentiability of the isochron excluding boundaries, and directional differentiability of the isochron within boundaries. This property is shown to be true in [56], justifying Assumptions 1-6. The studies diverge at this point: [56] reformulates the classic phase reduction approach of Kuramoto [31] as a Filippov system, allowing the application of classic nonsmooth theory. They then analyze the effects of weak, periodic forcing on oscillator entrainment. Our derivation of the jump matrix $M$ and its relation to the saltation matrix employs a first principles approach independent of the classic nonsmooth theory. We then consider examples of weakly coupled oscillators (as opposed to periodically forced oscillators), and we provide a rudimentary derivation of the interaction function (3.11) for weakly coupled oscillators with Filippov dynamics.

Other results that derive iPRCs or use the saltation matrix to determine stability of coupled oscillators cover special cases (the most common cases are a combination of planar systems, PWL systems, and relaxation oscillators). In cases in which the vector field is continuous across the switching boundary (but not differentiable across it) the saltation and jump matrices are trivial, and can be neglected. This situation holds in the original PWL version of the Fitzhugh-Nagumo model studied by McKean [40] and later incorporated into network models [8].

In [10], the authors analyze a network of PWL integrate-and-fire models with adaptation. They calculate the discontinuities of the iPRC using a method equivalent to the saltation matrix approach. The phase-locking analysis of this paper does not require the 
coupling strength to be small and the solutions are discontinuous, but requires all-to-all linear coupling between the planar oscillators. In contrast, our result requires continuous solutions and allows for nonlinear coupling of $n$-dimensional oscillators.

In some instances, properties of the LC solution allow a direct derivation of the iPRC using the normalization condition $(2.17)$ [7,26]. In these papers, the systems produce discontinuous solutions in the relaxation limit. The size of the discontinuity, along with the normalization condition, determines the discontinuous iPRC.

The saltation matrix is more commonly used to derive equations distinct from the iPRC, but with similar goals in mind. In [9], the authors derive the master stability function for PWL planar oscillators using the saltation matrix as part of their derivation. They use the saltation matrix to determine the Floquet exponents, which determine stability of oscillators in a synchronous state. Again, coupling between oscillators may be strong, but the coupling is linear and the oscillators are required to be identical and the stability analysis only holds for determining stability of the synchronous state. The authors remark, however, that deriving a master stability function for nonlinear coupling and higher dimensional models is possible in future work.

\subsection{Limitations of the method}

Our results apply broadly, because many systems have the structure we discuss. Nevertheless some caveats are in order.

The iPRC does not always capture the response to a stimulus. In situations in which the linear approximation to the asymptotic phase function breaks down, for instance when the stimulus drives the oscillator's trajectory close to the stable manifold of a saddle point on the boundary of the basin of attraction, mechanisms such as shear-induced chaos can lead to complicated responses to periodic forcing that cannot be predicted via iPRC analysis. This scenario can arise near the homoclinic bifurcation in the MorrisLecar model, for example [35]. In the limit that the periodic orbit is pushed towards a heteroclinic bifurcation the iPRC can diverge ( $c f$. Figures 6, 7), an effect discussed in [55]. Nevertheless, in many systems the iPRC plays an important role in understanding oscillator entrainment and synchronization.

In the examples we consider, the decomposition of the vector field into PWL domains is specified in the statement of the original system. The approximation of LCs in a smooth system, with LCs in a PWL system, has been investigated in a general setting [60]. However, there is no a priori heuristic for how to approximate an arbitrary nonlinear system with a PWL approximation.

Our method requires the phase function to be defined within some open neighbourhood of the LC (Assumption 4). For some nonsmooth systems, it is possible that the phase function may not be well defined throughout the entire basin of attraction of the LC. For example, Simpson and Jeffrey discuss piecewise smooth systems with a 'two-fold singularity' as a mechanism for finite time desynchronization of a LC oscillator [57]. In their example, the basin of attraction includes regions admitting sliding solutions that remain on the boundary surface for finite times, leading to nonuniqueness of solutions, and hence nonuniquness of the phase function in a portion of the stable manifold of the LC, (although uniqueness is guaranteed within a neighbourhood of the LC by the 
transverse flow condition). Our construction does not apply to such regions of the basin of attraction.

\subsection{Further applications}

The extension of results from classical dynamical systems to nonsmooth systems is an active area of research with applications in a wide variety of contexts. Carmona et al. studied a canonical form for LCs in planar PWL dynamical systems with two regions [5], using Melnikov methods to study existence and bifurcations of LCs. Ponce et al. studied bifurcations leading to LCs in PWL planar systems in [51]. Existence of LCs has been shown for planar PWL systems with two regions in [24] and [36], for planar PWL systems with an arbitrary but finite number of separate regions in [62], and for a PWL system in $\mathbb{R}^{4}$ with three regions in [6]. Stability of PWL LCs in $\mathbb{R}^{n}$ with $m+1$ regions is analyzed in [33] using Poincaré map techniques.

The review [34] discusses necessary and sufficient conditions for asymptotic stability of PWL systems in $\mathbb{R}^{n}$; [38] adapts Lyapunov functions for PWL systems.

LCs in PWL systems occur not only in biology but also in control engineering [48]. PWL systems arise naturally in anti-lock braking systems [43], which are themselves engineered to produce LC oscillations [49]. Piecewise smooth relay feedback systems, first used in heating [23] and more recently in (PID) control [29], can give rise to LCs. The exact conditions for LC existence in relay feedback systems is given in [61]. Many PWL biological models exist as well. A PWL version of the Fitzhugh-Nagumo model (also called the McKean model) and a PWL version of the Morris-Lecar model are studied in [8]. The authors in [3] convert the Hindmarsch-Rose model into a PWL version and analyze its stability. Gene regulatory networks are a classic example of PWL models exhibiting LC oscillations [19], and a subject of ongoing research. For instance, [12] analyzes the stability of synchronous periodic solutions, assuming weak symmetric coupling of two Glass networks. Rigorous investigations of Glass networks have considered them within the framework of differential inclusions [1,39]. To facilitate construction of networks with customized dynamics [64], systematically classified cyclic attractors on Glass networks with up to six switching units. Walsh and colleagues studied periodic orbits in a discontinuous vector field as a model of cycling phenomena in glacial dynamics [63]. In summary, there is a rich collection of contexts in which PWL and piecewise smooth systems arise.

\section{Conclusion}

The iPRC provides a linear approximation to the geometry of the asymptotic phase function in the vicinity of a stable LC. The classical method for obtaining iPRCs from the adjoint [15] breaks down with nonsmooth dynamics because the Jacobian may not be well defined at the domain boundaries. In this paper, we have introduced a general theory for the iPRCs for LCs arising in piecewise smooth systems, provided the LC intersects the domain boundaries transversely, the boundaries are smooth at the points of intersection, and that classic properties of the phase function exist at least within a boundary of the LC (Assumptions 1-6). In the case of piecewise smooth systems, which are also continuous 
across the domain boundaries, we obtain continuous iPRCs. Discontinuities in the iPRCs may arise when the vector field is discontinuous across domain boundaries, and our analysis provides the explicit form of the discontinuity, in the form of a linear matching condition related to the classical saltation matrix construction. Our results are consistent with, and extend, existing findings of iPRC calculations, such as [8]. Because piecewise smooth and PWL systems arise in a wide variety of fields, from biology to engineering, our analysis has the potential for broad application.

\section{Acknowledgements}

The authors thank Dr Yangyang Wang for suggesting several improvements to the manuscript.

\section{References}

[1] Acary, V., De Jong, H. \& Brogliato, B. (2014) Numerical simulation of piecewise-linear models of gene regulatory networks using complementarity systems. Physica D: Nonlinear Phenom. 269, 103-119.

[2] Aizerman, M. \& Gantmakher, F. (1958) On the stability of periodic motions. J. Appl. Math. Mech. 22(6), 1065-1078.

[3] Bizzarri, F., Linaro, D. \& Storace, M. (2007) PWL approximation of the Hindmarsh-Rose neuron model in view of its circuit implementation. In: Proceedings of the 18th European Conference on Circuit Theory and Design, 2007, IEEE, pp. 878-881.

[4] Brown, E., Moenlis, J. \& Holmes, P. (2004) On the phase reduction and response dynamics of neural oscillator populations. Neural Comput. 16(4), 673-715.

[5] Carmona, V., Fernández-García, S., Freire, E. \& Torres, F. (2013) Melnikov theory for a class of planar hybrid systems. Physica D: Nonlinear Phenom. 248, 44-54. http://www. sciencedirect.com/science/article/pii/S0167278913000067

[6] Cheng, Y. (2013) Bifurcation of limit cycles of a class of piecewise linear differential systems in with three zones. Discrete Dyn. Nat. Soc. 2013, Art. no. 385419.

[7] Coombes, S. (2001) Phase locking in networks of synaptically coupled McKean relaxation oscillators. Physica D: Nonlinear Phenom. 160(3), 173-188.

[8] Coombes, S. (2008) Neuronal networks with gap junctions: A study of piecewise linear planar neuron models. SIAM J. Appl. Dyn. Syst. 7, 1101-1129. http://link. aip.org/link/?SJA/ $7 / 1101 / 1$

[9] Coombes, S. \& Thul, R. (2016) Synchrony in networks of coupled non-smooth dynamical systems: Extending the master stability function. Eur. J. Appl. Math. 27(6), 904-922.

[10] Coombes, S., Thul, R. \& Wedgwood, K. (2012) Nonsmooth dynamics in spiking neuron models. Physica D: Nonlinear Phenom. 241(22), 2042-2057.

[11] Di Bernardo, M., Budd, C., Champneys, A. R. \& KowalczyK, P. (2008) Piecewise-Smooth Dynamical Systems: Theory and Applications, Applied Mathematical Sciences, Vol. 163, Springer Science \& Business Media, London.

[12] Edwards, R. \& Gill, P. (2003) On synchronization and cross-talk in parallel networks. Dynamics of Continuous Discrete and Impulsive Systems Series B 10, 287-300.

[13] Ermentrout, B. (1996) Type I membranes, phase resetting curves, and synchrony. Neural Comput. 8(5), 979-1001.

[14] Ermentrout, G. B. \& Terman, D. H. (2010) Foundations of Mathematical Neuroscience, Springer, Berlin, Germany.

[15] Ermentrout, G. \& Kopell, N. (1991) Multiple pulse interactions and averaging in systems of coupled neural oscillators. J. Math. Biol. 29(3), 195-217. http://dx.doi.org/10.1007/ BF00160535 
[16] Field, R. J. \& Noyes, R. M. (1974) Oscillations in chemical systems. IV. Limit cycle behavior in a model of a real chemical reaction. J. Chem. Phys. 60(5), 1877-1884.

[17] Filippov, A. F. (1988) Differential Equations with Discontinuous Righthand Sides, Mathematics and Its Applications, Kluwer Academic Publishers, Dordrecht, The Netherlands.

[18] Fruth, F., JÜlicher, F. \& Lindner, B. (2014) An active oscillator model describes the statistics of spontaneous otoacoustic emissions. Biophys. J. 107(4), 815-824.

[19] Glass, L. \& Pasternack, J. S. (1978) Stable oscillations in mathematical models of biological control systems. J. Math. Biol. 6, 207-223.

[20] Glass, L. \& Pérez, R. (1974) Limit cycle oscillations in compartmental chemical systems. $J$. Chem. Phys. 61(12), 5242-5249.

[21] Guckenheimer, J. (1975) Isochrons and phaseless sets. J. Math. Biol. 1, 259-273. 10.1007/BF01273747. http://dx.doi.org/10.1007/BF01273747

[22] Guckenheimer, J. \& Holmes, P. (1990) Nonlinear Oscillations, Dynamical Systems, and Bifurcations of Vector Fields, Applied Mathematical Sciences, 3rd ed., Vol. 42, Springer-Verlag, Berlin, Germany.

[23] Hawkins, J. (1887) Automatic regulators for heating apparatus. Trans. Amer. Soc. Mech. Eng. 9, 432 .

[24] HuAn, S.-M. \& YANG, X.-S. (2012) On the number of limit cycles in general planar piecewise linear systems. Discrete Continuous Dyn. Syst. 32(6), 2147-2164.

[25] IJSPEERT, A. J. (2008) Central pattern generators for locomotion control in animals and robots: A review. Neural Netw. 21(4), 642-53.

[26] Izhikevich, E. M. (2000) Phase equations for relaxation oscillators. SIAM J. Appl. Math. 60(5), 1789-1804.

[27] Izhikevich, E. M. (2007) Dynamical Systems in Neuroscience: The Geometry of Excitability and Bursting, Computational Neuroscience, MIT Press, Cambridge, Massachusetts.

[28] Izhikevich, E. M. \& Ermentrout, B. (2008) Phase model. Scholarpedia 3(10), 1487.

[29] Johansson, K. H., Barabanov, A. E. \& Åström, K. J. (2002) Limit cycles with chattering in relay feedback systems. IEEE Trans. Autom. Control 47(9), 1414-1423.

[30] Kelso, J. S., Holt, K. G., Rubin, P. \& Kugler, P. N. (1981) Patterns of human interlimb coordination emerge from the properties of non-linear, limit cycle oscillatory processes: theory and data. J. Motor Behavior 13(4), 226-261.

[31] Kuramoto, Y. (2003) Chemical Oscillations Waves and Turbulence, Dover, Mineola, New York, USA.

[32] Leine, R. \& NiJmeijer, H. (2004) Dynamics and Bifurcations of Non-Smooth Mechanical Systems, Vol. 18, Springer-Verlag, Heidelberg, Germany.

[33] Lin, C., Wang, Q.-G. \& Lee, T. H. (2003) Local stability of limit cycles for MIMO relay feedback systems. J. Math. Anal. Appl. 288(1), 112-123.

[34] Lin, H. \& Antsaklis, P. J. (2009) Stability and stabilizability of switched linear systems: a survey of recent results. IEEE Trans. Autom. Control 54(2), 308-322.

[35] Lin, K. K., Wedgwood, K. C. A., Coombes, S. \& Young, L.-S. (2012) Limitations of perturbative techniques in the analysis of rhythms and oscillations. J. Math. Biol 66, 139-161.

[36] Llibre, J. \& Ponce, E. (2012) Three nested limit cycles in discontinuous piecewise linear differential systems with two zones. Dyn. Contin. Discrete Impuls. Syst. Ser. B Appl. Algorithms 19(3), 325-335.

[37] Lyttle, D. N., Gill, J. P., Shaw, K. M., Thomas, P. J. \& Chiel, H. J. (2017) Robustness, flexibility, and sensitivity in a multifunctional motor control model. Biol. Cybern. 111(1), 25-47.

[38] Ma, Y., Yuan, R., Li, Y., Ao, P. \& Yuan, B. (2013) Lyapunov functions in piecewise linear systems: From fixed point to limit cycle. arXiv:1306.6880.

[39] Machina, A. \& Ponosov, A. (2011) Filippov solutions in the analysis of piecewise linear models describing gene regulatory networks. Nonlinear Anal.: Theor. Methods Appl. 74(3), 882-900. 
[40] McKean, H. (1970) Nagumo's equation. Adv. Math. 4, 209-223.

[41] Modolo, J., Henry, J. \& Beuter, A. (2008) Dynamics of the subthalamo-pallidal complex in Parkinsonõs disease during deep brain stimulation. J. Biol. Phys. 34(3-4), 251-266.

[42] Morrison, K., Degeratu, A., Itskov, V. \& Curto, C. (2016) Diversity of emergent dynamics in competitive threshold-linear networks: A preliminary report. arXiv:1605.04463 .

[43] Monse, A. (1997) Control Using Logic Based Switching, Lecture Notes in Control and Information Sciences, Vol. 222.

[44] Nowotny, T. \& Rabinovich, M. I. (2007) Dynamical origin of independent spiking and bursting activity in neural microcircuits. Phys. Rev. Lett. 98, 128106.

[45] Osinga, H. M. \& Moehlis, J. (2010) Continuation-based computation of global isochrons. SIAM J. Appl. Dyn. Syst. 9(4), 1201-1228.

[46] Park, Y. (2013) Infinitesimal Phase Response Curves for Piecewise Smooth Dynamical Systems, Master's Thesis, Case Western Reserve University.

[47] Park, Y. \& Ermentrout, B. (2016) Weakly coupled oscillators in a slowly varying world. $J$. Comput. Neurosci. 40(3), 269-281.

[48] Pettit, N. B. (1996) Analysis of Piecewise Linear Dynamical Systems, John Wiley \& Sons, New York, New York, USA.

[49] Pettit, N. B. \& Wellstead, P. E. (1995) Analyzing piecewise linear dynamical systems. Control Systems, IEEE 15(5), 43-50.

[50] Poggi, T., Sciutto, A. \& Storace, M. (2009) Piecewise linear implementation of nonlinear dynamical systems: From theory to practice. Electron. Lett. 45(19), 966-967.

[51] Ponce, E., Ros, J. \& Vela, E. (2013) The focus-center-limit cycle bifurcation in discontinuous planar piecewise linear systems without sliding. In: S. Ibáñez, J. S. Pérez del Río, A. Pumariño \& J. Á. Rodríguez (editors), Progress and Challenges in Dynamical Systems, Springer Proceedings in Mathematics and Statistics, Vol. 54, Springer, Berlin Heidelberg, pp. 335-349. http://dx.doi.org/10.1007/978-3-642-38830-9_21

[52] Rohden, M., Sorge, A., Timme, M. \& Witthaut, D. (2012) Self-organized synchronization in decentralized power grids. Phys. Rev. Lett. 109(6), 064101.

[53] Schwemmer, M. A. \& Lewis, T. J. (2012) The Theory of Weakly Coupled Oscillators. In: Phase Response Curves in Neuroscience Theory, Experiment, and Analysis, Springer Series in Computational Neuroscience, Vol. 6, Springer, pp. 3-31.

[54] Shaw, K. M., Lyttle, D. N., Gill, J. P., Cullins, M. J., McManus, J. M., Lu, H., Thomas, P. J. \& CHIEL, H. J. (2015) The significance of dynamical architecture for adaptive responses to mechanical loads during rhythmic behavior. J. Comput. Neurosci. 38(1), 25-51.

[55] Shaw, K. M., Park, Y., Chiel, H. J. \& Thomas, P. J. (2012) Phase resetting in an asymptotically phaseless system: On the phase response of limit cycles verging on a heteroclinic orbit. SIAM J. Appl. Dyn. Syst. 11(1), 350-391.

[56] Shirasaka, S., Kurebayashi, W. \& Nakao, H. (2017) Phase reduction theory for hybrid nonlinear oscillators. Phys. Rev. E 95(1), 012212.

[57] Simpson, D. J. W. \& Jeffrey, M. R. (2016) Fast phase randomization via two-folds. Proc. R. Soc. A: Math. Phys. Eng. Sci. 472(2186), 20150782.

[58] So, P., Francis, J. T., Netoff, T. I., Gluckman, B. J. \& Schiff, S. J. (1998) Periodic orbits: a new language for neuronal dynamics. Biophys. J. 74(6), 2776-2785.

[59] Stensby, J. L. (1997) Phase-Locked Loops: Theory and Applications, CRC Press, Boca Raton, Florida.

[60] Storace, M. \& De Feo, O. (2004) Piecewise-linear approximation of nonlinear dynamical systems. IEEE Trans. Circuits Syst. I: Regular Papers 51(4), 830-842.

[61] Tsypkin, I. Z. (1984) Relay Control Systems, Cambridge University Press, Cambridge, UK.

[62] G.Aiko, V. A. \& van Horssen W. T. (2009) Global analysis of a piecewise linear Liénard-type dynamical system. Int. J. Dyn. Syst. Differ. Equ. 2(1-2), 115-128.

[63] Walsh, J., Widiasih, E., Hahn, J. \& McGehee, R. (2016) Periodic orbits for a discontinuous vector field arising from a conceptual model of glacial cycles. Nonlinearity 29(6), 1843. 
[64] Zinovik, I., Chebiryak, Y. \& Kroening, D. (2010) Periodic orbits and equilibria in Glass models for gene regulatory networks. IEEE Trans. Inform. Theory 56(2), 805-820.

\section{Appendix A Proofs of the main results}

\section{A.1 Proof of Theorem 2.2}

From assumptions $1-3, \gamma$ is a piecewise smooth LC that admits a phase function $\theta(\boldsymbol{x})$ throughout the basin of attraction (B.A.), such that $d \theta / d t=1 / T$ throughout the B.A. Also, by assumption, $\theta$ is differentiable $\left(C^{1}\right)$ in the interior of each region and continuous $\left(C^{0}\right)$ at the boundaries between successive regions (assumptions 4 and 5).

For a piecewise smooth dynamical system satisfying hypotheses $\mathrm{H} 1-\mathrm{H} 4$ and assumptions $1-2$, the crossing point $\boldsymbol{p}_{k+1}$ lies in an $n-1$ dimensional surface $\Sigma_{k+1}$ that is $C^{1}$ within a ball $B\left(\boldsymbol{p}_{k+1}, c\right)$ of radius $c$, and admits a unique unit length normal vector $\hat{\boldsymbol{n}}$ oriented in the direction of the flow. By Gram-Schmidt, we may construct $n-1$ orthonormal vectors $\hat{\boldsymbol{w}}_{i}$ spanning the hyperplane tangent to $\Sigma_{k+1}$ at $\boldsymbol{p}_{k+1}$ orthogonal to $\hat{\boldsymbol{n}}$ for all $i=1, \ldots, n-1$.

Introduce local coordinates $u$ on $B\left(\boldsymbol{p}_{k+1}\right) \cap \Sigma_{k+1}$ such that $u=0$ corresponds to the point $\boldsymbol{p}_{k+1}$. Let $\theta_{k}$ denote the phase function $\theta$ within the $k$ th domain. Although the gradient $\nabla \theta$ is not defined at points on the boundary $\Sigma_{k+1}$, we have well defined directional derivatives

$$
\begin{aligned}
D_{\mathbf{w}}\left(\theta_{k}\right)\left(\mathbf{x}_{k}\right) & =\lim _{h \rightarrow 0} \frac{1}{h}\left(\theta\left(\mathbf{x}_{k}+h \mathbf{w}\right)-\theta\left(\mathbf{x}_{k}\right)\right) \\
D_{\mathbf{w}}\left(\theta_{k+1}\right)\left(\mathbf{x}_{k+1}\right) & =\lim _{h \rightarrow 0} \frac{1}{h}\left(\theta\left(\mathbf{x}_{k+1}+h \mathbf{w}\right)-\theta\left(\mathbf{x}_{k+1}\right)\right),
\end{aligned}
$$

for points $\mathbf{x}_{k}$ and $\mathbf{x}_{k+1}$ in the interior of region $k$ and region $k+1$, respectively. Fixing a basis vector $\hat{\boldsymbol{w}}_{i}$ in the plane tangent to $\Sigma_{k+1}$ at $\boldsymbol{p}_{k+1}$ and taking the limits as $\mathbf{x}_{k} \rightarrow \boldsymbol{p}_{k+1}$ and $\mathbf{x}_{k+1} \rightarrow \boldsymbol{p}_{k+1}$, we have (Assumption 6)

$$
\hat{\boldsymbol{w}}_{i} \cdot \nabla \theta_{k+1}(u)=\hat{\boldsymbol{w}}_{i} \cdot \nabla \theta_{k}(u), \quad \forall i=1, \ldots, n-1,
$$

(for simplicity, we use $\hat{\boldsymbol{w}}_{i}$ in place of $\hat{\boldsymbol{w}}_{i}^{k+1}$ ). For $u=0$, the gradient of the phase function is evaluated on the LC. Referring back to the notation of equation (2.12) we have

$$
\hat{\boldsymbol{w}}_{i} \cdot z_{k+1,0}=\hat{\boldsymbol{w}}_{i} \cdot z_{k, t_{k}}, \quad \forall i=1, \ldots, n-1
$$

Equation (A 4) provides $n-1$ independent linear equations in the $n$ unknown values $z_{j}$, $j=1, \ldots n$. To obtain the $n$th independent linear equation, let $m \in\{k, k+1\}$ and let $\boldsymbol{x}_{m}(t)$ be a trajectory in the basin of attraction of vector field $\boldsymbol{F}_{m}$. By the chain rule,

$$
\begin{aligned}
\frac{d \theta_{m}}{d t} & =\left[\nabla \theta_{m}\left(\boldsymbol{x}_{m}(t)\right)\right] \cdot \frac{d}{d t} \boldsymbol{x}_{m}(t) \\
& =\boldsymbol{F}_{m}\left(x_{m}(t)\right) \cdot \nabla \theta_{m}\left(\boldsymbol{x}_{m}(t)\right) .
\end{aligned}
$$


For a trajectory $\boldsymbol{x}_{m}(t)$ on the LC, i.e., when $\boldsymbol{x}_{m}(t)=\gamma_{m}(t)$, we have by definition of the iPRC [14],

$$
\frac{d \theta_{m}}{d t}=\boldsymbol{F}_{m}\left(\gamma_{m}(t)\right) \cdot \boldsymbol{z}_{m}(t)
$$

Recalling that $d \theta / d t=1 / T$, taking the one-sided limits,

$$
\begin{gathered}
\lim _{t \rightarrow 0^{+}} \boldsymbol{F}_{k+1}\left(\gamma_{k+1}(t)\right) \cdot \boldsymbol{z}_{k+1}(t)=\boldsymbol{F}_{k+1,0} \cdot \boldsymbol{z}_{k+1,0}, \\
\lim _{t \rightarrow t_{k}^{-}} \boldsymbol{F}_{k}\left(\gamma_{k}(t)\right) \cdot \boldsymbol{z}_{k}(t)=\boldsymbol{F}_{k, t_{k}} \cdot \boldsymbol{z}_{k, t_{k}},
\end{gathered}
$$

therefore,

$$
\boldsymbol{F}_{k+1,0} \cdot z_{k+1,0}=\frac{1}{T}=\boldsymbol{F}_{k, t_{k}} \cdot z_{k, t_{k}} \cdot
$$

Equation (A 8) asserts that the phase function advances at the same rate as a function of time everywhere, and in particular on both sides of the boundary point $\boldsymbol{p}_{k+1}$. Combining equations (A 8) with (A 4) provides $n$ independent linear equations:

$$
\begin{aligned}
\boldsymbol{F}_{k+1,0} \cdot \boldsymbol{z}_{k+1,0} & =\boldsymbol{F}_{k, t_{k}} \cdot \boldsymbol{z}_{k, t_{k}}, \\
\hat{\boldsymbol{w}}_{1} \cdot \boldsymbol{z}_{k+1,0} & =\hat{\boldsymbol{w}}_{1} \cdot \boldsymbol{z}_{k, t_{k}}, \\
\hat{\boldsymbol{w}}_{2} \cdot \boldsymbol{z}_{k+1,0} & =\hat{\boldsymbol{w}}_{2} \cdot \boldsymbol{z}_{k, t_{k}}, \\
\vdots & \\
\hat{\boldsymbol{w}}_{n-1} \cdot \boldsymbol{z}_{k+1,0} & =\hat{\boldsymbol{w}}_{n-1} \cdot \boldsymbol{z}_{k, t_{k}},
\end{aligned}
$$

which are equivalent to the equality expressed in Theorem 2.2 in terms of the $n \times n$ matrices $C_{k+1}$ and $D_{k}$ as defined in the theorem.

In order to solve for the initial value of the iPRC of the $k+1$ portion of the LC, $z_{k+1,0}$, we must invert the matrix $C_{k+1}$. The invertibility of $C_{k+1}$ is guaranteed because the vector field value $\boldsymbol{F}_{k+1,0}$ and the $n-1$ tangent vectors are linearly dependent if and only if $\boldsymbol{F}_{k+1,0} \in \operatorname{span}\left(\hat{\mathrm{w}}_{1}, \ldots, \hat{\mathrm{w}}_{\mathrm{n}-1}\right)$. However, the vector $\boldsymbol{F}_{k+1,0}$ can not be written as a linear combination of every $\hat{\boldsymbol{w}}_{i}$, because the vector $\boldsymbol{F}_{k+1,0}$ is always transverse to the boundary at the point $\boldsymbol{p}_{k+1}$ by assumption. Therefore, the vector $\boldsymbol{F}_{k+1,0}$ and the $n-1$ orthonormal basis vectors of the tangent hyperplane at the point $\boldsymbol{p}_{k+1}$ are linearly independent, and the matrix $C_{k+1}$ is invertible.

The boundary crossing point considered in the proof is arbitrary, so the proof applies to all boundary crossings of the LC.

\section{A.2 Proof of Corollary 2.3}

We adopt the same assumptions as Theorem 2.2. By the assumptions stated in Section 2.1, the LC, $\gamma$, consists of $K$ distinct sections, each passing through a linear vector field $\boldsymbol{F}_{1}, \boldsymbol{F}_{2}, \ldots, \boldsymbol{F}_{K}$. The solution to the adjoint equation of the first region (equation (2.8)) is

$$
z_{1}(t)=e^{A_{1} t} \hat{z}_{1,0}
$$


where $A_{1}=-D F_{1}\left(\gamma_{1}(t)\right)^{T}$, the negative transpose of the Jacobian matrix $D F_{1}$ evaluated along the $\operatorname{LC} \gamma(t), e^{A_{1} t}$ is a matrix exponential, and $\hat{z}_{1,0}$ is an initial condition of the iPRC in the first region. Because the vector field is linear within each region, the Jacobian matrix is piecewise constant. The initial condition of the iPRC of the next region $z_{2,0}$ may be written in terms of the initial condition of the iPRC of the first region:

$$
\begin{aligned}
z_{2,0} & =M_{2} z_{1, t_{1}} \\
& =M_{2} e^{A_{1} t_{1}} \hat{z}_{1,0} .
\end{aligned}
$$

Similarly, the initial condition of the iPRC of the third region may be written as,

$$
\begin{aligned}
z_{3,0} & =M_{3} z_{2, t_{2}} \\
& =M_{3} e^{A_{2} t_{2}} M_{2} e^{A_{1} t_{1}} \hat{\mathbf{z}}_{1,0},
\end{aligned}
$$

and so forth. Upon traversing the $K$ th region we return to $\hat{z}_{1,0}$, which must satisfy

$$
\hat{z}_{1,0}=M_{1} e^{A_{K} t_{K}} \cdots M_{2} e^{A_{1} t_{1}} \hat{z}_{1,0}=: B \hat{z}_{1,0} .
$$

Therefore, $\hat{z}_{1,0}$ is a unit eigenvector of $B$. Uniqueness of the unit eigenvector (up to multiplication by a constant) follows from the stability of the LC (assumption 1). If $B \mathbf{q}=\mathbf{q}$ for another eigenvector $\mathbf{q} \notin \operatorname{span}\left(\hat{\mathbf{z}}_{1,0}\right)$, an arbitrarily small initial condition could be found near $\boldsymbol{p}_{1}$ that did not converge to $\gamma$.

Uniqueness of the magnitude of $\hat{z}_{1,0}$ comes from equation (2.17), which we recall to be

$$
\boldsymbol{F}_{1,0} \cdot z_{1,0}=\frac{1}{T}
$$

where $z_{1,0}$ is the unique initial condition of the iPRC of region 1 . The vector $\hat{z}_{1,0}$ must be scaled by some constant $\alpha$ to be equivalent to the initial condition, $z_{1,0}$. We can calculate the scaling by use of equation (A 14):

$$
\begin{aligned}
\boldsymbol{F}_{1,0} \cdot\left(\alpha \hat{z}_{1,0}\right) & =\frac{1}{T} \\
\alpha \boldsymbol{F}_{1,0} \cdot \hat{z}_{1,0} & =\frac{1}{T} \\
\alpha & =\frac{1}{T\left(\boldsymbol{F}_{1,0} \cdot \hat{z}_{1,0}\right)} .
\end{aligned}
$$

Thus, the unique initial condition of the iPRC, $z_{1,0}$ is

$$
\begin{aligned}
z_{1,0} & =\alpha \hat{z}_{1,0} \\
& =\frac{\hat{z}_{1,0}}{T\left(\boldsymbol{F}_{1,0} \cdot \hat{z}_{1,0}\right)} .
\end{aligned}
$$

This concludes the proof of uniqueness for the initial condition of the iPRC for affine linear vector fields satisfying Theorem 2.2. 


\section{A.3 Proof of Corollary 2.4}

Let $t$ denote global time, $T_{k}=\sum_{j=1}^{k} t_{j}$ where $t_{k}$ is the time of flight for region $k$, $(1 \leqslant k \leqslant K)$, and $A_{k}=-\left(D F_{k}\right)^{T}$, where $D F_{k}$ is the Jacobian matrix of the vector field $\boldsymbol{F}_{k}$. Beginning with region 1 , the affine linear vector field assumption allows us to explicitly solve for the iPRC using matrix exponentials. We determine the initial condition of region $1, z_{1,0}$, using Corollary 2.3. Then, for any $0 \leqslant t<T_{1}$,

$$
z(t)=e^{A_{1} t} z_{1,0}
$$

This iPRC solution reaches the next boundary at time $t_{1}$, and takes the value $z\left(t_{1}^{-}\right)=$ $e^{A_{1} t_{1}} z_{1,0}$. To continue the solution into the next region, we compute the jump matrix $M_{2}$ using Theorem 2.2 and apply it to the value $z\left(t_{1}^{-}\right)$to place the iPRC solution on the initial condition on the boundary of region $2, z_{2,0} \equiv M_{2} e^{A_{1} t_{1}} z_{1,0}$. Using this initial condition, and for any $T_{1}<t<T_{2}$, we compute the iPRC solution to be

$$
z(t)=e^{A_{2}\left(t-T_{1}\right)} z_{2,0} \equiv e^{A_{2}\left(t-T_{1}\right)} M_{2} e^{A_{1} t_{1}} z_{1,0} .
$$

By repeating this process into the final region $K$, the solution results in an alternating product of jump matrices and matrix exponentials for any $T_{K-1}<t<T_{K}$ :

$$
z(t)=e^{A_{k}\left(t-T_{K-1}\right)} M_{K} e^{A_{K-1} t_{K-1}} \cdots M_{2} e^{A_{1} t_{1}} z_{1,0}
$$

This equation completes the proof.

Remark A.1 Because the iPRC solution is periodic and isolated, continuing this process from region $K$ into region 1 results in the solution

$$
\begin{aligned}
z(t) & =e^{A_{1} t} M_{K} e^{A_{k}\left(t_{K}\right)} \cdots M_{2} e^{A_{1} t_{1}} z_{1,0} \\
& =e^{A_{1} t} B z_{1,0},
\end{aligned}
$$

for $n T \leqslant t<n T+T_{1}$, for any integer $n$. The last line is a trivial substitution that follows from Corollary 2.3.

\section{A.4 Proof of Corollary 2.6}

If the vector fields are of class $C^{0}$ over boundaries, then the vector field coordinates $F^{+}$and $F^{-}$(see equation (2.14)) are equal. Therefore, the matrices $C_{k+1}$ and $D_{k}$ of equation (2.14) are identical, and because $M_{k+1}=C_{k+1}^{-1} D_{k}$, the matrix $M_{k+1}$ reduces to the identity matrix for each $k$. 


\section{Appendix B Calculation details for the nominal biting model of Aplysia califonica}

Recall equation (3.4)

$$
\frac{d \boldsymbol{r}}{d t}=\left\{\begin{array}{c}
1-x-(y+a) \rho \\
y+a, \quad x \geqslant y+a, x \geqslant z-a, \\
(z-a)(1-\rho) \\
(x-a)(1-\rho) \\
1-y-(z+a) \rho, \quad y>x-a, y \geqslant z+a, \\
z+a \\
x+a \\
(y-a)(1-\rho), \quad z>x+a, z>y-a, \\
1-z-(x+a) \rho
\end{array}\right.
$$

In the domain, the three corners given by the standard basis vectors each have a saddle point. Because the vector field is linear within each region, the stable manifold is a plane spanned by the two stable eigenvectors of the Jacobian for each region, and the unstable manifold is the (half) line in the direction of the unstable eigenvector. The two stable eigenvectors and the unstable eigenvector for region 1 are, respectively,

$$
(1,0,0),(0,0,1),\left(-\frac{\rho}{2}, 1,0\right) \text {. }
$$

The vectors for the saddle in region 2 are, respectively,

$$
(0,1,0),(1,0,0),\left(0,-\frac{\rho}{2}, 1\right)
$$

and for region 3 ,

$$
(0,0,1),(0,1,0),\left(1,0,-\frac{\rho}{2}\right)
$$

When $a>0$ the heteroclinic cycle is broken, and the unstable manifold of each vector field $\boldsymbol{F}_{k}$ flows into the boundary surface between vector fields $\boldsymbol{F}_{k}$ and $\boldsymbol{F}_{k+1}$ (as opposed to flowing along the boundary edge when $a=0$, where there is a nonempty intersection of the unstable manifold of $\boldsymbol{F}_{k}$ and the stable manifold of $\boldsymbol{F}_{k+1}$ ). The vector fields $\boldsymbol{F}_{1}, \boldsymbol{F}_{2}$, and $\boldsymbol{F}_{3}$, are shifted by vectors $s_{1}, s_{2}$, and $s_{3}$, respectively, where

$$
\begin{aligned}
& s_{1}=(0,-a, a), \\
& s_{2}=(a, 0,-a), \\
& s_{3}=(-a, a, 0) .
\end{aligned}
$$

As in the example of the modified iris system, the LC of this PWL stable heteroclinic cycle model is not analytically computable. The time of flight for each portion of the LC, $t_{k}$, must be derived numerically for each $k$ and for fixed parameter values $a$ and $\rho$. The LC coordinates are obtained numerically; we denote them $\boldsymbol{p}_{k+1}=\left(\eta_{k+1}, \kappa_{k+1}, v_{k+1}\right)$, 
i.e., for the $k$ th portion of the LC, its initial value is the vector $\gamma_{k, 0}=\left(\eta_{k}, \kappa_{k}, v_{k}\right)$. We now calculate the jump matrices $M_{k+1}$ for each boundary $\boldsymbol{p}_{k+1}$, beginning with region 1 .

The normal vector at $\boldsymbol{p}_{2}$ and its two tangent vectors, $\hat{\boldsymbol{u}}_{2}$ and $\hat{\boldsymbol{w}}_{2}$, are

$$
\begin{aligned}
& \hat{\boldsymbol{n}}_{2}=\left(-\frac{1}{\sqrt{2}}, \frac{1}{\sqrt{2}}, 0\right), \\
& \hat{\boldsymbol{u}}_{2}=(0,0,1), \\
& \hat{\boldsymbol{w}}_{2}=\left(-\frac{1}{\sqrt{2}},-\frac{1}{\sqrt{2}}, 0\right) .
\end{aligned}
$$

By Theorem 2.2, the jump matrix $M_{2}$ is

$$
M_{2}=\left(\begin{array}{ccc}
a_{11} & a_{12} & a_{13} \\
a_{21} & a_{22} & -a_{13} \\
0 & 0 & a_{33}
\end{array}\right)
$$

where

$$
\begin{aligned}
& a_{11}=\eta_{2}+(\rho-1) \kappa_{2}-\rho v_{2}, \\
& a_{12}=1-a(1+\rho)-2 \kappa_{2}-\rho v_{2}, \\
& a_{13}=-a(\rho-2)+\rho v_{2}, \\
& a_{21}=1+a(1-2 \rho)+(\rho-2) \eta_{2}-\rho \kappa_{2}, \\
& a_{22}=\kappa_{2}-a(\rho-2)+(\rho-1) \eta_{2}, \\
& a_{33}=1+a(1-2 \rho)+(\rho-1) \eta_{2}-\kappa_{2}-\rho .
\end{aligned}
$$

The remaining jump matrices are

$$
M_{3}=\left(\begin{array}{ccc}
b_{11} & 0 & 0 \\
b_{21} & b_{22} & b_{23} \\
-b_{21} & b_{32} & b_{33}
\end{array}\right)
$$

where

$$
\begin{aligned}
& b_{11}=a(2 \rho-1)+\rho \eta_{3}-(\rho-1) \kappa_{3}+v_{3}-1, \\
& b_{21}=a(\rho-2)-\rho \eta_{3}, \\
& b_{22}=\rho \eta_{3}-\kappa_{3}-(\rho-1) v_{3}, \\
& b_{23}=a(\rho+1)+\rho \eta_{3}+2 v_{3}-1, \\
& b_{32}=a(2 \rho-1)-(\rho-2) \kappa_{3}+\rho v_{3}-1, \\
& b_{33}=a(\rho-2)-(\rho-1) \kappa_{3}-v_{3},
\end{aligned}
$$

and

$$
M_{1}=\left(\begin{array}{ccc}
c_{11} & c_{12} & c_{13} \\
0 & c_{22} & 0 \\
c_{31} & -c_{12} & c_{33}
\end{array}\right)
$$


where

$$
\begin{aligned}
& c_{11}=a(\rho-2)-\eta_{1}-(\rho-1) v_{1}, \\
& c_{12}=\rho \kappa_{1}-a(\rho-2), \\
& c_{13}=2 \rho a-a+\rho \eta_{1}-(\rho-2) v_{1}-1, \\
& c_{22}=2 \rho a-a+\eta_{1}+\rho \kappa_{1}-\rho v_{1}+v_{1}-1, \\
& c_{31}=\rho a+a+2 \eta_{1}+\rho \kappa_{1}-1, \\
& c_{33}=-(\rho-1) \eta_{1}+\rho \kappa_{1}-v_{1} .
\end{aligned}
$$

The solution to the adjoint equation for each region is

$$
\begin{aligned}
& z_{1}(t)=\left(\begin{array}{ccc}
e^{t} & 0 & 0 \\
\rho \sinh (t) & e^{-t} & 0 \\
0 & 0 & e^{t(\rho-1)}
\end{array}\right) z_{1,0}, \\
& z_{2}(t)=\left(\begin{array}{ccc}
e^{t(1-\rho)} & 0 & 0 \\
0 & e^{t} & 0 \\
0 & \rho \sinh (t) & e^{-t}
\end{array}\right) z_{2,0}, \\
& z_{3}(t)=\left(\begin{array}{ccc}
e^{-t} & 0 & \rho \sinh (t) \\
0 & e^{t(\rho-1)} & 0 \\
0 & 0 & e^{t}
\end{array}\right) z_{3,0} .
\end{aligned}
$$

